\title{
Direct $N$-body Simulations of Rubble Pile Collisions
}

\author{
Zoë M. Leinhardt, Derek C. Richardson, and Thomas Quinn \\ Department of Astronomy, University of Washington, Box 351580, Seattle, Washington 98195-1580 \\ E-mail: zoe@astro.washington.edu
}

Received August 18, 1999; revised January 11, 2000

\begin{abstract}
There is increasing evidence that many kilometer-sized bodies in the Solar System are piles of rubble bound together by gravity. We present results from a project to map the parameter space of collisions between kilometer-sized spherical rubble piles. The results will assist in parameterization of collision outcomes for Solar System formation models and give insight into disruption scaling laws. We use a direct numerical method to evolve the positions and velocities of the rubble pile particles under the constraints of gravity and physical collisions. We test the dependence of the collision outcomes on impact parameter and speed, impactor spin, mass ratio, and coefficient of restitution. Speeds are kept low $\left(<10 \mathrm{~m} \mathrm{~s}^{-1}\right.$, appropriate for dynamically cool systems such as the primordial disk during early planet formation) so that the maximum strain on the component material does not exceed the crushing strength, assuming sufficient granularity. We compare our results with analytic estimates and hydrocode simulations. We find that net accretion dominates the outcome in slow head-on collisions while net erosion dominates for fast off-axis collisions. The dependence on impact parameter is almost equally as important as the dependence on impact speed. Off-axis collisions can result in fast-spinning elongated remnants or contact binaries while fast collisions result in smaller fragments overall. Clumping of debris escaping from the remnant can occur, leading to the formation of smaller rubble piles. In the cases we tested, less than $2 \%$ of the system mass ends up orbiting the remnant. Initial spin can reduce or enhance collision outcomes, depending on the relative orientation of thespin and orbital angular momenta. We derivea relationship between impact speed and angle for critical dispersal of mass in the system. We find that our rubble piles are relatively easy to disperse, even at low impact speed. This may provide a way of constraining the energy dissipation parameter and related properties of the initial planetesimal population. (c) 2000 A cademic Press
\end{abstract}

Key Words: asteroids; collisional physics; impact processes; planetary formation; planetesimals.

\section{INTRODUCTION}

There is growing interest in understanding the dynamics of collisions between small bodies in the Solar System. Typically such collisions are divided into two regimes: those dominated by material strength and those dominated by self-gravity (Holsapple 1994). The transition from the strength to the gravity regime may occur at body sizes as small as a few kilometers for basalt (Ryan and Melosh 1998, Benz and Asphaug 1999) or as small as $250 \mathrm{~m}$ for silicates (Love and Ahrens 1996). In this paper we present numerical results from simulations of collisions in the gravity regime. Our experiments are primarily concerned with low-speed collisions between equal-mass, kilometer-sized "rubble piles," gravitationally bound aggregates of loose material. We believe that these experiments will shed light on the collisional dynamics of the protoplanetary disk when typical encounter speeds are comparable to the surface escape speed (about $1 \mathrm{~m} \mathrm{~s}^{-1}$ for kilometer-sized planetesimals of $2 \mathrm{~g} \mathrm{~cm}^{-3}$ bulk density)

\subsection{Definitions}

We begin with definitions of terms frequently encountered in the context of binary collision experiments. Typically in the literature one impactor (the larger one) is stationary and is considered to be the target, while the other (the smaller one) is moving and is called the projectile. In our experiments, the impactors are comparable in size and are both in motion, so we generally do not distinguish between a target and a projectile. Most laboratory experiments involve solid targets that possess tensile strength, so the outcome is measured in terms of the extent of disruption or shattering of the target. A critical or catastrophic shattering event is one in which the largest post-impact fragment (the remnant) has $50 \%$ of the target mass. Following a recently adopted convention in the literature (Durda et al. 1998), we use $Q_{\mathrm{S}}^{*}$ to denote the kinetic energy per unit target mass to achieve critical shattering. A rubble pile, by definition, has no tensile strength, so $Q_{\mathrm{S}}^{*}$ is effectively zero. However, a rubble pile can still be disrupted in the sense that one or more of the component particles becomes separated from the rest for at least an instant.

For collisions in free space, fragments or particles are said to be dispersed if they attain positive orbital energy with respect to the remnant. Hence, a critical or catastrophic dispersal is one in which the largest remnant is left with $50 \%$ of the original target mass after the remaining material has dispersed to infinity. The energy per unit target mass to achieve this is denoted by $Q_{\mathrm{D}}^{*}$. In our experiments, since we do not distinguish between a target and a projectile, $Q_{\mathrm{D}}^{*}$ refers to the energy per unit total mass, in 
the center-of-mass frame, needed to critically disperse the entire system.

Finally, we define erosion to mean permanent removal of mass from a body, and accretion to mean permanent retention of mass. In the context of our experiments, net erosion means that one body (the largest if the impactors are of unequal mass) had less mass at the end of the run than it started with. Net accretion means it had more mass at the end.

\subsection{Motivation}

Many asteroid characteristics are inconsistent with monolithic configurations. Recent observations by the Near Earth Asteroid Rendezvous spacecraft of Mathilde, a 53-km C-class asteroid, are particularly suggestive. First, Mathilde's largest crater is enormous: it has a diameter of $33.4 \mathrm{~km}$, almost $7 \mathrm{~km}$ larger than the asteroid's mean radius (Veverka et al. 1997). Numerical hydrocode simulations and laboratory experiments strongly suggest that in order for Mathilde to have survived the impact that formed such a substantial crater, the asteroid must be made of some material that does not efficiently transmit energy throughout the body (Love et al. 1993, Asphaug et al. 1998, Housen et al. 1999). Second, Mathilde has a remarkably low density of $1.3 \mathrm{~g} \mathrm{~cm}^{-3}$ (Yeomans et al. 1997), about one-third the average value for the chondritic meteorites that are thought to originate from C-class asteroids (Wasson 1985). Such a low density suggests that Mathilde is highly porous. If true, the voids in the material could impede the transmission of energy from a collisional shock wave and allow a rather weak body to survive an otherwise catastrophic impact event. We also note the recent discovery of the asteroid satellite S/1998 (45) 1, which implies a density of $\sim 1.2 \mathrm{~g} \mathrm{~cm}^{-2}$ for the main body Eugenia (Merline et al. 1999).

In addition to Mathilde, the surfaces of 243 Ida, 951 Gaspra, and Phobos show several sizable craters that have diameters on the order of the mean radius of the body (for references, see Richardson et al. 1998, hereafter Paper I). As in the case of Mathilde, the energy necessary to create craters of this size would disperse or disrupt the original body if it were solid (Asphaug and Melosh 1993).

Further evidence for the prevalence of rubble piles comes from asteroid spins. In a sample of 107 asteroids smaller than $10 \mathrm{~km}$ in diameter, Harris (1996) found that the spin period distribution truncates at fast spin rates, where rubble piles would start to fly apart. ${ }^{1}$

One explanation for the observed characteristics of these asteroids and their craters is that they are rubble piles. Although rubble pile configurations are more susceptible to disruption by tidal forces than monolithic configurations (Paper I), there is increasing evidence that rubble piles have a higher impact strength (Ryan et al. 1991, Love and Ahrens 1996, Asphaug et al. 1998).

\footnotetext{
${ }^{1}$ At least one asteroid spinning faster than this limit has since been discovered (Ostro et al. 1999), but its small size ( $\sim 30 \mathrm{~m}$ ) puts it comfortably in the strength regime.
}

There are two scenarios for creating a rubble-pile asteroid: (1) the asteroid is initially one solid body of material and is rubblized over time by multiple impacts; (2) the rubble-pile configuration of the asteroid is primordial. Regardless of how rubble-pile asteroids are formed it is interesting to investigate how they interact and evolve in the Solar System. In addition to asteroids there is a considerable amount of evidence that a large percentage of comet nuclei are rubble piles, for example, the tidal disruption of Comet D/Shoemaker-Levy 9 (Richardson et al. 1995, Asphaug and Benz 1996).

\subsection{Laboratory Experiments: Strength vs Gravity}

Ryan et al. (1991) presented results from a laboratory study of impacts into weak inhomogeneous targets. Due to practical limitations they used $\sim 0.5-\mathrm{cm}$ targets of gravel and glue. As a result, their specific experimental results are firmly rooted in the strength regime. However, the most general conclusion that the group arrived at from dropping, crashing, and shooting at the gravel aggregates was that the relatively weak targets have a surprisingly high impact strength. In other words, it took a large amount of energy (at least $Q_{\mathrm{S}}^{*}=40 \mathrm{~J} \mathrm{~kg}^{-1}$ ) to critically disrupt or shatter the target such that the largest remnant was one-half the mass of the original object. The nonuniformity of the target causes a greater fraction of the impact energy to dissipate thermally; therefore, the collisional shock wave is more efficiently absorbed by the target.

Laboratory experiments on Earth to investigate directly the collisional dynamics of the gravity regime are difficult to conduct since the target size necessary to reach this regime is impractically large. Instead, overpressure and centrifuge techniques have been used to artificially simulate the gravity regime in the laboratory. In an overpressure experiment, Housen et al. (1991) used nitrogen gas at various pressures to mimic the lithostatic stress felt inside a large target. At these pressures they were unable to carry out true impact tests, so they used a buried charge instead of a projectile. As the pressure was increased, the size of the largest remnant after each explosion also increased, indicating a transition from the strength-dominated regime to the pressuredominated regime. Housen et al. (1991) argued that the pressure regime was analogous to the gravity regime and extrapolated a scaling law for the gravity regime from the overpressure data. This laboratory study has two important drawbacks: (1) by using a buried charge the experiment does not model the actual surface dynamics of an asteroid during an impact; (2) the gas overpressure is not an $r^{-2}$ force law. They were able to reach a regime in the laboratory that was not dominated by the strength of the material, but it is unclear whether the gravity-regime scaling law derived from the overpressure data is valid.

In a centrifuge experiment, Housen et al. (1999) were able to conduct true impact tests by firing a small projectile (a polyethylene cylinder $0.65 \mathrm{~cm}$ in diameter) from a gas gun strapped to the arm of a centrifuge. They positioned a porous target (composed of quartz sand, perlite, fly ash, and water) at the end of the arm. The centrifuge was used to mimic the gravitational force at the 
surface of a much larger body. The use of the centrifuge introduces second-order complexities due to the Coriolis force and the field orientation in general at the surface of the cylindrical target (though this is only really a problem in the event of high ejecta trajectories). In addition, the flat surface of the target may subtly affect crater morphology. Nonetheless, this experiment showed that porous targets in the gravity regime are efficient at absorbing impact energy at the surface by compacting the underlying material.

\subsection{Numerical Simulations of Collisions and Tidal Disruptions}

Extrapolations of laboratory experiments have resulted in rough strength and gravity scaling laws. In order to truly understand the collisional dynamics and evolution of large bodies, numerical simulations are a necessity. For example, Love and Ahrens (1996) used a three-dimensional "smoothed particle hydrodynamics" (SPH) code to simulate high-speed catastrophic collisions. They used various impact speeds $\left(3-7 \mathrm{~km} \mathrm{~s}^{-1}\right)$, impact angles $\left(5-75^{\circ}\right)$, target diameters $(10-1000 \mathrm{~km})$, and projectile diameters $(0.8-460 \mathrm{~km})$ in order to explore a large region of parameter space. The big targets placed the experiments securely in the gravity regime, allowing the researchers to treat gravity carefully and neglect the strength and fracturing of the target completely. Their extrapolated scaling law for the gravity regime placed the transition from the strength to the gravity regime at a target diameter of $250 \pm 150 \mathrm{~m}$, much smaller than that predicted by laboratory experiments (Holsapple 1994). Love and Ahrens (1996) argue that since smaller asteroids are more common than larger ones, a given asteroid is more likely to suffer a shattering impact before a dispersing impact. Thus, it seems plausible that many asteroids in our Solar System are at least partial rubble piles.

More recent simulations have had similar results. Asphaug et al. (1998) conducted three high-speed $\left(5 \mathrm{~km} \mathrm{~s}^{-1}\right)$ impact experiments using a solid target, a partially rubblized contact binary, and a totally rubblized target. In each case the researchers used a small projectile six orders of magnitude less massive than the target. There are three major conclusions from this study: (1) it is much easier to disrupt a solid target than it is to disperse it - this conclusion is evidence that it is possible to change a solid body into a rubble pile with impacts; (2) rubble regions can insulate and block energy from traveling through a body-in a contact binary, for example, one end could be critically disrupted while the other remains undamaged; (3) the fully rubblized targets efficiently localize the energy transmitted during a collision which in turn minimizes the damage outside the collision region and allows weak bodies to survive high-energy impacts with much less damage than solid targets. This again implies that many small bodies in the Solar System may be rubble piles. Other similar numerical experiments include Ryan and Melosh (1998) and Benz and Asphaug (1999).

Watanabe and Miyama (1992) used 3D SPH code to investigate the effects of tidal distortion and shock compression from collisional impacts in the process of planetary accumulation.
They used two equal-sized spherical bodies and assumed a perfect Newtonian fluid. It is important to note that their code did not model an incompressible fluid (their adopted polytropic indices were always greater than zero). As a result of experimenting with impact angle, speed, and density gradients, they found that tidal forces can enlarge the coalescence rate of planetesimals by almost a factor of 2 . In addition, when the initial speed of the impactor is significantly lower than the escape speed of the system, less than a few percent of the total mass is lost from the system in the collision. They did not attempt any simulations with initial speeds in excess of $50 \%$ of the escape speed.

In Paper I, Richardson et al. numerically simulated the effects of Earth's tidal force on rubble-pile asteroids. Unlike Watanabe and Miyama (1992), they simulated the Earth-crossing asteroids as incompressible fluids using a hard-sphere model. They varied the asteroids' speed, spin, shape, and close-approach distance. Generally, slow-moving, close-approaching, prograde-rotating, elongated asteroids were the most susceptible to tidal disruption. They found several distinct classes of outcome: in the most violent disruption cases, the asteroid was stretched into a line and recollapsed into a "string of pearls" reminiscent of Comet D/Shoemaker-Levy 9 at Jupiter; for moderate disruptions, large pieces of the asteroid were stripped off in many cases, forming satellites or contact binaries; the mildest disruptions resulted in little mass loss but significant shape changes. These various outcomes could lead to the formation of crater chains (Bottke et al. 1997), asteroid satellites and doublet craters (Bottke and Melosh 1996a,b), and unusually shaped asteroids (Bottke et al. 1999).

Durda (1996) carried out simulations to study how readily satellites form as a result of mutual gravitational attraction after the catastrophic disruption of the progenitor. Durda (1996) came to three major conclusions: (1) satellites do form immediately after a catastrophic collision; (2) contact binaries form more easily than true binary systems; (3) the binary systems form in a wide range of size ratios. It is important to realize that Durda (1996) assumed a power-law mass distribution for the catastrophically fragmented asteroid. The slope index used (1.833) was taken from extrapolations of laboratory experiments.

\subsection{Implications for Planet Formation}

Traditionally, numerical simulations of planet formation use extrapolations of impact experiments in the strength regime to model the effects of fragmentation in planetesimal collisions (e. g., Greenberg et al. 1978, Beaugé and Aarseth 1990, Wetherill and Stewart 1993). From what we have already seen, such extrapolations may give misleading results since generally much more energy is needed to disperse than to disrupt a planetesimal in the gravity regime. Moreover, effects of impact angle, spin, and impactor mass ratio are often not taken into account. In the case of rubble piles, no empirical model actually exists. For example, we might expect reaccumulation like that seen in the tidal disruption models to also occur after the catastrophic impact of two rubble-pile planetesimals. In this paper we aim to explore these issues by simulating collisions between rubble-pile bodies 
over a wide range of parameter space and determining the implications of the results for planet formation. In Section 2 we describe our numerical method and analysis technique. Our results are presented in Section 3, followed by a general discussion in Section 4. We give our conclusions in Section 5.

\section{METHOD}

The simulation and analysis of the collisions presented here combine numerical methods introduced in Paper I and Richardson et al. (1999, hereafter Paper II). The rubble pile model is an extension of the model used for studying the tidal disruption of asteroids (Paper I). The integration engine is an extension of the parallel tree code used for planetesimal evolution simulations (Paper II).

\subsection{Rubble Pile Model}

Each rubble pile in our simulations consists, at least initially, of a fixed number of equal-size hard spheres arranged in "hexagonal close-packed" (HCP) form. The rubble piles are typically generated by specifying the bulk semi-axes, bulk density, and approximate number of particles (alternatively, the particle radius and/or density can be used as independent parameters). The generator attempts to match the requested properties on the basis of the estimated HCP efficiency of a sphere as a function of bulk radius or number of particles (derived from power-law fits to our own numerical experiments). Once the rubble pile is constructed, the constituent particles are reduced in size by a fixed factor (usually $1 \%$ ) and given a small random velocity kick (no more than $10 \%$ of the particle surface escape speed in magnitude). This is to facilitate attaining the initial equilibrium (cf. Section 2.4). Finally, the rubble pile is tagged with a unique "color" so that mixing can be studied visually and statistically.

The collisional properties of the constituent particles are specified prior to each simulation. These include the normal and tangential coefficients of restitution, $\epsilon_{\mathrm{n}}$ and $\epsilon_{\mathrm{t}}$ (cf. Richardson 1994). Except for certain explicit test models, these values generally were fixed at $\epsilon_{\mathrm{n}}=0.8$ (mostly elastic collisions with some dissipation) and $\epsilon_{\mathrm{t}}=1.0$ (no surface friction). Bouncing was the only possible collision outcome: no mergers or fragmentations of particles were allowed. The value of $\epsilon_{\mathrm{n}}$ was chosen to be consistent with Paper I and is similar to experimentally determined values used in the literature (e.g., Beaugé and Aarseth 1990). Note that in the perfectly elastic case, particles cannot recollapse into condensed rubble piles after a disruption event but instead completely disperse or at best end up in centrally concentrated swarms. In the case of tidal disruption (Paper I) the outcome is relatively insensitive to the choice of $\epsilon_{\mathrm{n}}$, so long as $\epsilon_{\mathrm{n}}<1$. For the present study, however, varying $\epsilon_{\mathrm{n}}$ has a stronger effect, an issue we explore in Section 3.2. We did not include surface friction in the present study, in order to keep the number of test cases manageable.

There are two circumstances under which $\epsilon_{\mathrm{n}}$ is allowed to change. First, if the relative speed of two colliding particles is less than $10 \%$ of their mutual escape speed (i.e., typically $\sim 1 \mathrm{~cm} \mathrm{~s}^{-1}$ ), $\epsilon_{\mathrm{n}}$ is set to unity (no dissipation). This is to prevent computationally expensive "sliding motions" (Petit and Hénon 1987). Second, if the collision speed exceeds $10 \mathrm{~m} \mathrm{~s}^{-1}, \epsilon_{\mathrm{n}}$ is set to 0.2 (highly dissipative). This is to crudely model damping through internal fracture as the impact stress $\rho v c$ ( $\rho=$ internal density, $c=$ sound speed $\sim 10^{3} \mathrm{~m} \mathrm{~s}^{-1}$ ) exceeds the "rock" strength $\left(\sim 10^{7} \mathrm{~N} \mathrm{~m}^{-2}\right)$. This is not intended to be a physically rigorous model but rather a simple mechanism to prevent unrealistically high collision speeds. Initial encounter speeds between rubble piles were generally kept closer to $1 \mathrm{~m} \mathrm{~s}^{-1}$ in any case. Also, particle sizes were kept roughly comparable across rubble piles in order to minimize any strength-versus-size biases.

It is important to note that neither rolling nor true sliding motions are modeled in our code. Moreover, particles cannot remain mutually at rest in contact (i.e., there are no surface normal forces). Instead, the constituent particles of an otherwise quiescent rubble pile are in a constant state of low-energy collisional vibration (dictated by the minimum sliding condition described above). Nevertheless, such small bounces can mimic transverse motions in an approximate sense in the presence of shear flow, giving realistic bulk properties to the material. To test this we have simulated the formation of sand piles using our collision code (with surface friction) that give reasonable values for the angle of repose when compared with laboratory experiments.

\subsection{Numerical Code}

Our simulations were performed using a modified version of a cosmological $N$-body code, pkdgrav (Stadel and Quinn, in preparation; data structures described in Anderson 1993, 1996). This is a scalable, parallel tree code designed for ease of portability and extensibility. For the parameter space study, the parallel capability was not exploited owing to the modest number of particles in each run (a few thousand). However, even in serial mode, pkdgrav is arguably more efficient than any other existing code with similar capability. In particular, it is superior to box_tree, the code used in Paper I, which could handle only a few hundred particles in practical fashion.

A low-order leapfrog scheme is used as the pkdgrav integrator. The comparative simplicity of this scheme is a big advantage for collision prediction since particle position updates are linear in the velocity term. This means that every possible collision within the time step can be determined in advance and in the correct sequence. Time steps are smaller than in higher-order schemes for the same accuracy, but the cost of each gravity calculation is far outweighed by the collision search once the rubble piles are in contact and is comparable otherwise. Moreover, away from collision, particle trajectories are integrated symplectically, eliminating spurious numerical dissipation. For further detail and references, refer to Paper II.

Although the collision search is relatively expensive, the scaling is modest: $\mathcal{O}(N \log N)$ with particle number and linear with the number of collisions per interval. A typical encounter between thousand-particle rubble piles can generate $\sim 10^{8}$ 
collisions over the course of a run! A balanced $k$-d tree (Bentley and Friedman 1979) is used to search for possible collisions at the beginning of each time step, giving the $\mathcal{O}(N \log N)$ dependence. Once a collision is performed, only particles that might be affected by the event in the remaining interval (numbering typically $\ll N)$ are reconsidered via the neighbor search, giving the near linear dependence on the number of collisions. This latter enhancement is an improvement to the Paper II code, which did not require as much sophistication given the low collision frequency per step. Note that the collision search can also be performed in parallel, which proved necessary for the large- $N$ models presented in Section 3.3 below.

\subsection{Hardware}

The parameter space models were run on a local cluster of 16 300-MHz Intel Pentium IIs using the High Throughput Computing (HTC) environment condor (cf. http://www . cs.wisc.edu/condor/) under RedHat Linux. The condor system supports automatic scheduling, submission, and restarting of jobs on shared resources, greatly simplifying management. A typical run required between 12 and 72 wallclock hours to complete and each generated $\sim 25-50 \mathrm{MB}$ of data. Models requiring parallel resources were run either on a local cluster of four 433-MHz DEC Alpha PCs connected with a fast ethernet switch, or on a local SGI Origin 200 with four $180-\mathrm{MHz}$ processors running IRIX. Both platforms typically achieved sustained performances of several hundred megaflops.

\subsection{Initial Conditions}

Generation of initial conditions and analysis of results were performed using code auxiliary to pkdgrav. The rubble pile generator has already been described (Section 2.1). Each new rubble pile was first run in isolation (with or without spin) using pkdgrav until the velocity dispersion of the constituent particles achieved a stable equilibrium. Next a new "world" was created by using a small program to position and orient any number of equilibrated rubble piles (always two in the present study) prior to simulation. Spherical bodies were usually given a random orientation in order to reduce the effect of HCP planes of symmetry. Bulk velocities were then applied to each rubble pile. Other rubble pile properties that could be changed at this point included the total mass, bulk radius, bulk density, and color. For the exploration of parameter space, usually only the positions (in the form of $y$ offsets), velocities, spins, and colors were modified. Once all the rubble piles were in place, the world was adjusted so that the center of mass coincided with the origin and the velocity of the center of mass was zero. The output world was then read in by pkdgrav and the simulation would begin.

To facilitate the exploration of parameter space, a series of Unix scripts were written to generate and monitor each run. Starting with a given pair of rubble piles and a list of desired initial impact parameters, speeds, and spins, the world generator was run automatically to create the necessary initial condi- tions and support files in separate run directories. The scheduler condor was then invoked to farm the jobs out to all available machines. Analysis was performed on the fly using a machine outside the condor pool for maximum efficiency.

The choice of initial conditions was governed largely by prior test simulations. For the parameter space exploration, 10 values of impact parameter $b$ and 10 values of initial relative speed $v$ were chosen for each set of runs, where a set consisted of a fixed choice of spin and/or offset direction (see Section 3 for a complete description of each model). From the test simulations it was clear that only about half of the possible 100 runs for each model were needed to find the representative cases and the $Q_{\mathrm{D}}^{*}$ boundary. In a plot of $b$ vs $v$, the important region is the lowerleft triangle (see Fig. 2 for an example). The $b$ and $v$ values were therefore chosen to sample this region as finely as possible in a practical amount of time. Models with spin were chosen to sample representative combinations of spin and orbital angular momentum at a fixed rotation period.

\subsection{Coordinate System and Units}

We use an inertial Cartesian coordinate system in free space for our simulations, with the origin at the center of mass. In the parameter space studies, the initial motion of the colliding bodies is in the $\pm x$ direction. Any initial impact parameter is measured in the $\pm y$ direction. Most debris actually travels in directions perpendicular to the original axis of motion (cf. Section 4.3).

A natural unit for the impact parameter $b$ is the sum of the radii $R_{1}+R_{2}$ of the two (spherical) impactors. Hence $b=0$ implies a head-on collision while $b=1$ is a grazing encounter. Note, however, the true trajectories will generally be hyperbolae; no allowance is made for this in the definition. Since tidal effects may play an unpredictable role anyway, we adopt the simpler definition. In the absence of trajectory deflection, the impact angle is then $\phi=\sin ^{-1} b$, for $b \leq 1$.

The unit for the initial relative speed $v$ is more complicated. We chose a system in which $v=0$ indicates no relative motion and $v=1$ is the estimated critical speed for dispersal. The critical speed is found by equating the initial total kinetic energy with the gravitational binding energy of a rubble pile made up of a spherical and homogeneous mixture of both colliders,

$$
v_{\text {crit }}=M \sqrt{\frac{6 G}{5 \mu R}},
$$

where $M$ is the combined mass, $G$ is the gravitational constant, $\mu$ is the reduced mass $M_{1} M_{2} / M$, and $R$ is the radius of the sphere that contains the combined mass, assuming the same bulk density:

$$
R=\left(R_{1}^{3}+R_{2}^{3}\right)^{1 / 3}
$$

Note that the actual speed at impact will slightly exceed $v$ due to gravitational acceleration. 
In the parameter space models, the initial separation in $x$ for all cases was $\sim 6 R$, effectively 2.5 Roche radii for the combined mass, i.e., far enough apart that initial tidal effects were negligible. The total energy of the system was positive in all cases. For completeness, the speed at infinity is given by

$$
v_{\infty}=\left(v^{2} v_{\text {crit }}^{2}-\frac{G M \cos \phi}{3 R}\right)^{1 / 2}
$$

and the speed at impact is

$$
v_{\text {impact }}=\left(v_{\infty}^{2}+\frac{2 G M}{R_{1}+R_{2}}\right)^{1 / 2} .
$$

\subsection{Run Parameters}

Most pkdgrav run parameters assumed default values for these simulations (cf. Paper II). However, in addition to the collision parameters described in Section 2.1, the run time, time step, and output frequency were specified explicitly for each model.

The run time $\left(t_{\mathrm{r}}\right)$ was initially 10 times the characteristic time,

$$
t_{\mathrm{c}} \sim \sqrt{\frac{x^{3}}{G M}}
$$

where $x$ is the initial separation. Typically $t_{\mathrm{r}}$ is $\sim 36 \mathrm{~h}$. In most cases this is sufficient time for the postcollision system to reach a steady state. Some cases were run longer (typically a factor of 2) if necessary, on the basis of visual inspection of animations.

The time step for each run was set to a small value $t_{0}$ times a heuristic scale factor of $1 /(2 v+1)$, arrived at by trial and error from our test runs (recall that $v$ is the initial speed, so $t_{0}$ is a simple constant). The scaling ensures finer intervals for neighbor searches in higher-speed impacts (this is necessary to avoid missing any potential collisions). For our runs, $t_{0}=$ $10^{-5}$ year $/ 2 \pi$, or roughly $50 \mathrm{~s}$. Note that for objects with bulk density a few $\mathrm{g} \mathrm{cm}^{-3}$ the dynamical time $1 / \sqrt{G \rho} \sim 1 \mathrm{~h}$, comfortably large compared to the maximum adopted time step. Generally our simulations are limited by the time needed to deal with particle collisions, so the gravity calculations can be of higher accuracy with little additional cost.

Finally, the output frequency was chosen so that there would be about 200 outputs per run, suitable for smooth animations and analysis.

\subsection{Analysis Method}

Much of our analysis method is similar to that presented in Paper I; the reader is referred to that work for additional details. The basic strategy is to identify the largest postcollision remnant, compute its various properties, and generate statistics for the relative distribution of the smaller fragments. We use a slightly different clump-finding algorithm (Section 2.7.1) and now employ a shape drawing technique (Section 2.7.2). We have made other refinements that should improve the accuracy of the analysis.
2.7.1. Clump finding. The clump-finding algorithm iteratively refines gusses as to what constitutes a rubble pile by merging groups of particles together in bottom-up fashion. The first guess is that every particle in the system is its own rubble pile. On each pass basic properties are computed for each clump: mass, position, axis lengths, and orientation. Clumps are then compared in pairwise fashion. In order for two clumps to be merged (i.e., to be considered one clump), spheres of diameter equal to the major axes times a fixed linking scale (a dimensionless number $>1$, typically 1.1) and centered on each clump must overlap. If the scaled minor axes also overlap, then the clumps are merged. Failing that, if either body has its center of mass in the other's scaled ellipsoid, the bodies are merged. Otherwise, no merge occurs. This process iterates until there are no more mergers during an iteration.

This method is purely geometrical: gravitational groupings are not considered. This was done mostly because there is no natural gravitational length scale in the present context, unlike in Paper I where the Hill radius could be used. However, osculating elements of groups measured with respect to the largest fragment are still calculated and give a good indication of the future evolution of the system. The present method also differs from Paper I in that it treats each clump as an ellipsoid rather than a sphere, allowing more refined boundaries to be drawn. The linking scale of 1.1 was adopted through trial and error (visual inspection).

2.7.2. Shape drawing. During the course of the present investigation we came across some unusual, often asymmetrical shapes following collision events. In order to characterize these forms, a shape-drawing algorithm was devised. The algorithm attempts to trace the outer surface of a given rubble pile (either in cross section or by projection to the $x-y$ plane). The resulting shape is equivalent to what would be measured by laser beams aimed at the surface in the direction of the center of mass. Note that this means that any outcroppings can conceal underlying structure. Generally such complex surfaces are not seen in our models, however (as confirmed by 3D VRML viewing). The projection method is used in the parameter space plots of Section 3.

2.7.3. Mixing. The unique color assigned to each rubble pile makes it easy to assess visually the degree of mixing following a collision. In order to make a more quantitative assessment, we have constructed the following statistic,

$f_{\text {mix }}=1-\frac{1}{\sqrt{N_{v}}} \sum_{c}\left[\sum_{v}\left(\frac{m_{c, v}}{\sum_{c^{\prime}} m_{c^{\prime}, v}}-\frac{m_{c, \text { world }}}{\sum_{c^{\prime}} m_{c^{\prime}, \text { world }}}\right)^{2}\right]^{1 / 2}$,

where subscript $c$ denotes a color, subscript $v$ denotes a subvolume of the rubble pile, $N_{v}$ is the number of subvolumes, and "world" refers to the entire population of particles in the system. Note that particle number is conserved so that $\sum_{c} m_{c \text {, world }}=M$, the total mass of the system. This formula is generalized for any number of components (colors); in the present study only two 
populations were considered. A mixing fraction of unity implies the rubble pile contains a perfectly homogeneous mixture of the world colors. A value of zero means no mixing has taken place at all.

Spherical subvolumes are used to sample different regions of the rubble pile (which itself need not be spherical). The size of the sample region is set so that it contains $\sqrt{N}$ particles on average. The center of a subvolume is chosen randomly within a rectangular prism enclosing the rubble pile. A new subvolume is chosen if the region is found to contain fewer than $N^{1 / 4}$ particles. Otherwise, the argument of the $\sum_{v}$ in Eq. (6) is computed and added to the running sum. This is repeated until $\sqrt{N}$ subvolumes are successfully sampled.

\section{RESULTS}

We now present the results of our simulations. First we describe the parameter space exploration which consisted of numerous runs of modest size. Highlights are shown in Fig. 1, where we have endeavored to illustrate the various classes of outcomes. Second we show the dependence on the coefficient of restitution $\epsilon_{\mathrm{n}}$ for a particular high-energy run. Finally we present the results of two high-resolution cases and compare with the corresponding moderate-resolution runs.

\subsection{Parameter Space}

We divided our exploration of parameter space into three models: a generic case as a baseline, a case with spinning impactors, and a case with unequal-mass impactors. Graphical summaries of these models are given in Figs. 2 and 4, which are discussed in detail below.

3.1.1. Model A: Equal size, no spin. Model A, our generic case, consisted of two equal-size rubble piles of $1 \mathrm{~km}$ radius and $2 \mathrm{~g} \mathrm{~cm}^{-3}$ bulk density. The rubble piles were generated and equilibrated using the process described in Section 2.4. Each rubble pile contained 955 identical spherical particles of $83 \mathrm{~m}$ radius, so the packing efficiency was $55 \% .^{2}$ The parameter space extends from $0.00-1.25$ in $b$ and $0.52-2.50$ in $v$ (the units of $b$ and $v$ are defined in Section 2.5; $v_{\text {crit }}=2.06 \mathrm{~m} \mathrm{~s}^{-1}$ for this model). The impact parameter values were chosen to encompass a range of dynamic interactions from head-on collisions to glancing distortions. The lowest value of $v$ is twice the value corresponding to $v_{\infty}=0$ (cf. Eq. (3); smaller $v$ leads to strong trajectory deflections). The largest value of $v$ was chosen to be a moderately high-speed impact to ensure that the catastrophic dispersal regime was entered.

Figure 2 summarizes the results of this model (Figs. 1a-1c give snapshots of three distinct outcomes). The shapes in Fig. 2 trace the projected silhouettes of the largest post-encounter fragment at the end of each run. We have used nested squares of dif-

\footnotetext{
2 The effective packing efficiency is less than the maximum close-packed efficiency of $74 \%$ due to finite-size effects (Paper I).
}

ferent line styles to divide our results into three mass regimes. A solid-line inner square indicates that the largest fragment contains $90 \%$ or more of the total mass of the system, i.e., nearly perfect accretion. A dashed-line inner square indicates that the largest fragment contains at least $50 \%$ but less than $90 \%$ of the total mass. The remaining cases contain less than $50 \%$ of the total mass in the largest fragment, i.e., net erosion. Note if there is no mass loss or exchange during the encounter the largest fragment will contain $50 \%$ of the total mass of the system by definition. We see in this model that 18 of 55 runs (33\%) result in net mass loss, although we caution that several cases are just on the border of $50 \%$.

The general trends in Fig. 2 are twofold, namely, as the encounter speed increases, the size of the largest fragment decreases, and as the impact parameter increases, the axis ratio increases, up to a certain point. Higher encounter speeds imply larger kinetic energy so it is more likely for the system to become unbound. Larger impact parameters imply larger angular momentum which results in an increase in the axis ratio until the critical spin value of the combined rubble pile is reached (cf. Eq. (7)). In addition to the general trends, the middle-mass group has two distinct populations that reflect their formation history. The small $b$, large $v$ group (top left in the figure) represents a net loss of mass of 10-50\% from the system. The large $b$, small $v$ group (lower right) represents grazing collisions with little mass loss or exchange.

We note that for the head-on case our definition of $v_{\text {crit }}$ does not correspond to critical dispersal, rather, critical dispersal seems to occur at $\sim 1.9 v_{\text {crit }}(\sim 4$ times the binding energy of the rubble pile). This probably reflects the fact that the energy of the collision is not immediately transported to all of the particles and that the voids in between the particles decrease the efficiency of energy propagation. Moreover, we did not take into account $\epsilon_{\mathrm{n}}$ in the definition of $v_{\text {crit }}$. Regardless, $v_{\text {crit }}$ is intended as an approximate scaling only.

More detailed results for this model are given in Table I. In the table, $b$ and $v$ have the usual definitions; $M_{\text {rem }}$ is the mass fraction of the largest postencounter remnant; $P$ is its instantaneous spin period in hours; $\varepsilon$ is the remnant's "ellipticity": $\varepsilon \equiv 1-$ $\frac{1}{2}\left(q_{2}+q_{3}\right)$, where $q_{2} \equiv a_{2} / a_{1}, q_{3} \equiv a_{3} / a_{1}$, and $a_{1} \geq a_{2} \geq a_{3}$ are the semi-axes ( $\varepsilon=0$ is a sphere); $f_{\text {mix }}$ is given by Eq. (6); $M_{\text {acc }}, M_{\text {orb }}$, and $M_{\text {esc }}$ are the mass fractions that are accreting, orbiting, and escaping from the largest remnant, respectively ${ }^{3}$; and $n_{1}, n_{2}$, and $n$ are the number of single particles, two-particle groups, and discrete rubble piles (i.e., groups with three or more particles), respectively, at the end of the run. The $M_{\text {rem }}$ column of Table I compliments Fig. 2 by providing a finer gradation of the remnant mass. Note that $M_{\text {rem }}+M_{\text {acc }}+M_{\text {orb }}+$ $M_{\mathrm{esc}} \equiv 1$.

\footnotetext{
${ }^{3}$ To be considered accreting, a clump must have $q<r+R$, where $q$ is the close-approach distance to the remnant, and $r$ and $R$ are the radii of minimal spheres enclosing the clump and remnant, respectively. This differs somewhat from Paper I.
} 

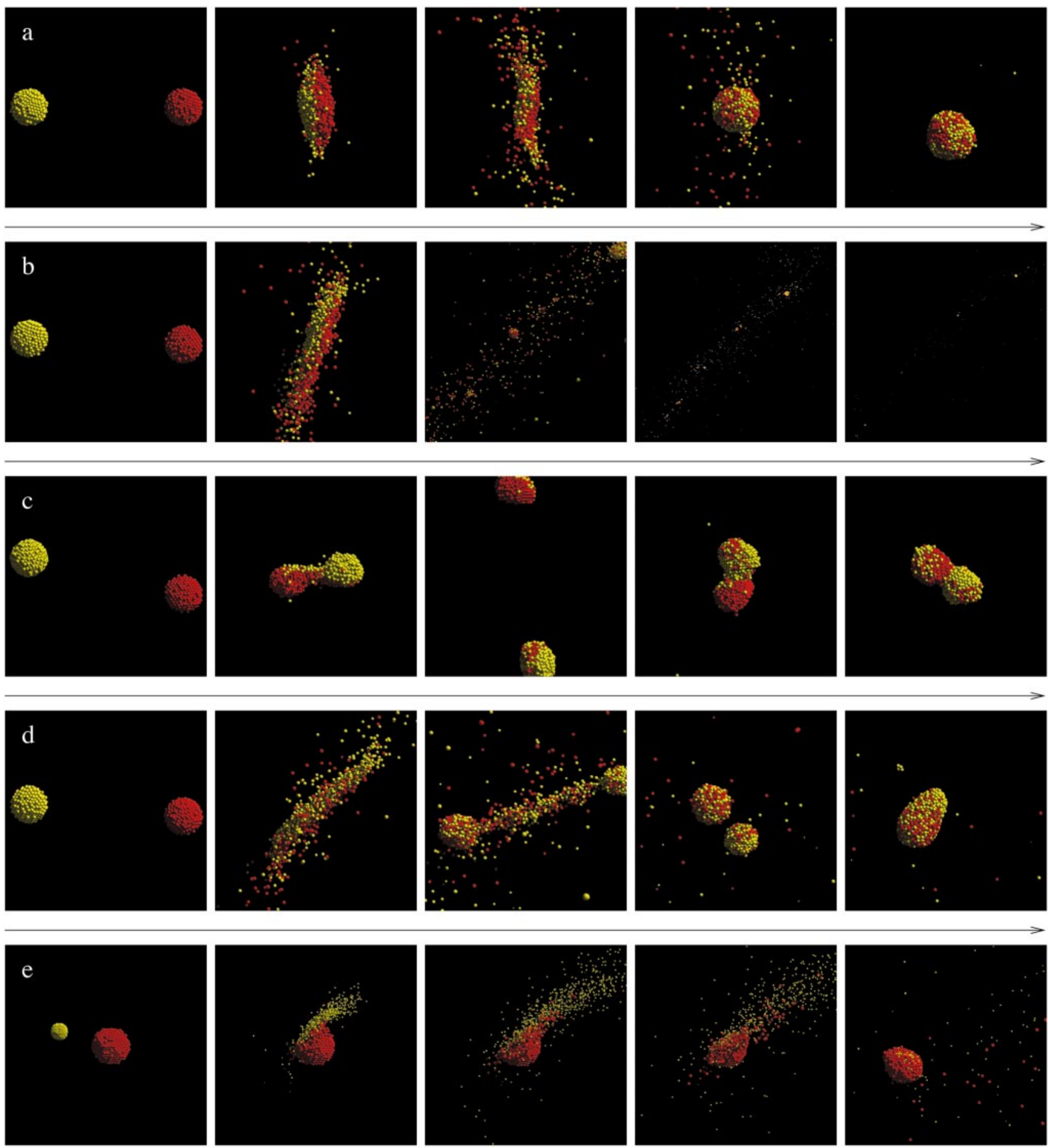

FIG. 1. Snapshots of rubble pile collisions from representative runs as seen in the center-of-mass frame. The models and runs are: (a) Model A, $b=0.00$, $v=1.00$; (b) Model A, $b=0.15, v=2.00$; (c) Model A, $b=0.90, v=0.52$; (d) Model B1, $b=0.30, v=1.10$; and (e) Model C, $b=0.50, v=1.25$. The arrow of time is to the right. The interval between frames is not regular: the snapshots were chosen to highlight distinct stages in the evolution of each run. In run (b), the final two frames have been brightened for clarity.

Table I shows how the remnant spin $P$ is coupled to the angular momentum in each run. Since there are no external torques in the system, angular momentum is conserved. In the case of head-on collisions $(b=0)$, there is exactly zero total angular momentum, which accounts for the large remnant $P$ values (i.e., low spin). $P$ is never infinite in these cases because some particles escape and carry angular momentum away from the remnant, even in the slowest collision case $(v=0.52)$. At higher collision speeds, 
TABLE I

Summary of Model A Results (Section 3.1.1)

\begin{tabular}{|c|c|c|c|c|c|c|c|c|c|c|c|}
\hline$b$ & $v$ & $M_{\mathrm{rem}}$ & $P$ & $\varepsilon$ & $f_{\text {mix }}(\%)$ & $M_{\mathrm{acc}}$ & $M_{\mathrm{orb}}$ & $M_{\mathrm{esc}}$ & $n_{1}$ & $n_{2}$ & $n$ \\
\hline 0.00 & 0.52 & 0.992 & 641.2 & 0.09 & $26 \pm 4$ & 0.001 & 0.000 & 0.007 & 15 & 0 & 1 \\
\hline 0.00 & 0.61 & 0.989 & 281.7 & 0.08 & $29 \pm 3$ & 0.001 & 0.000 & 0.010 & 21 & 0 & 1 \\
\hline 0.00 & 0.75 & 0.971 & 220.8 & 0.06 & $33 \pm 4$ & 0.004 & 0.000 & 0.025 & 55 & 0 & 1 \\
\hline 0.00 & 0.90 & 0.936 & 181.6 & 0.05 & $38 \pm 5$ & 0.007 & 0.000 & 0.057 & 114 & 4 & 1 \\
\hline 0.00 & 1.00 & 0.928 & 110.8 & 0.05 & $50 \pm 4$ & 0.007 & 0.000 & 0.065 & 134 & 2 & 1 \\
\hline 0.00 & 1.10 & 0.903 & 114.3 & 0.04 & $52 \pm 4$ & 0.007 & 0.000 & 0.090 & 182 & 2 & 1 \\
\hline 0.00 & 1.25 & 0.843 & 116.2 & 0.08 & $64 \pm 4$ & 0.013 & 0.000 & 0.145 & 285 & 4 & 3 \\
\hline 0.00 & 1.50 & 0.699 & 20.7 & 0.07 & $73 \pm 3$ & 0.022 & 0.000 & 0.279 & 531 & 11 & 7 \\
\hline 0.00 & 2.00 & 0.374 & 19.1 & 0.04 & $80 \pm 4$ & 0.046 & 0.016 & 0.564 & 938 & 39 & 27 \\
\hline 0.00 & 2.50 & 0.098 & 5.1 & 0.31 & $71 \pm 4$ & 0.004 & 0.007 & 0.891 & 1328 & 34 & 38 \\
\hline 0.15 & 0.52 & 0.992 & 11.9 & 0.07 & $25 \pm 3$ & 0.002 & 0.000 & 0.006 & 16 & 0 & 1 \\
\hline 0.15 & 0.61 & 0.984 & 11.0 & 0.14 & $28 \pm 3$ & 0.002 & 0.000 & 0.014 & 30 & 0 & 1 \\
\hline 0.15 & 0.75 & 0.965 & 9.1 & 0.12 & $30 \pm 4$ & 0.005 & 0.000 & 0.030 & 66 & 0 & 1 \\
\hline 0.15 & 0.90 & 0.944 & 7.4 & 0.14 & $37 \pm 4$ & 0.006 & 0.000 & 0.050 & 105 & 1 & 1 \\
\hline 0.15 & 1.00 & 0.924 & 6.9 & 0.12 & $40 \pm 4$ & 0.004 & 0.000 & 0.072 & 142 & 2 & 1 \\
\hline 0.15 & 1.10 & 0.885 & 6.1 & 0.13 & $47 \pm 4$ & 0.016 & 0.000 & 0.099 & 197 & 4 & 4 \\
\hline 0.15 & 1.25 & 0.818 & 5.7 & 0.07 & $59 \pm 3$ & 0.012 & 0.001 & 0.169 & 324 & 8 & 2 \\
\hline 0.15 & 1.50 & 0.695 & 5.6 & 0.09 & $59 \pm 3$ & 0.017 & 0.008 & 0.280 & 529 & 12 & 6 \\
\hline 0.15 & 2.00 & 0.275 & 8.4 & 0.04 & $52 \pm 5$ & 0.009 & 0.005 & 0.710 & 939 & 24 & 30 \\
\hline 0.30 & 0.52 & 0.994 & 6.9 & 0.20 & $21 \pm 3$ & 0.001 & 0.000 & 0.005 & 11 & 0 & 1 \\
\hline 0.30 & 0.61 & 0.988 & 6.0 & 0.20 & $28 \pm 3$ & 0.002 & 0.000 & 0.010 & 23 & 0 & 1 \\
\hline 0.30 & 0.75 & 0.974 & 5.3 & 0.22 & $34 \pm 3$ & 0.002 & 0.000 & 0.025 & 50 & 0 & 1 \\
\hline 0.30 & 0.90 & 0.946 & 4.6 & 0.20 & $37 \pm 3$ & 0.006 & 0.001 & 0.047 & 103 & 0 & 1 \\
\hline 0.30 & 1.00 & 0.901 & 4.4 & 0.24 & $37 \pm 4$ & 0.009 & 0.010 & 0.081 & 184 & 3 & 1 \\
\hline 0.30 & 1.10 & 0.887 & 4.5 & 0.41 & $40 \pm 4$ & 0.018 & 0.010 & 0.085 & 202 & 5 & 2 \\
\hline 0.30 & 1.25 & 0.786 & 4.5 & 0.42 & $42 \pm 4$ & 0.013 & 0.020 & 0.182 & 366 & 10 & 7 \\
\hline 0.30 & 1.50 & 0.408 & 7.6 & 0.09 & $32 \pm 4$ & 0.029 & 0.007 & 0.555 & 481 & 17 & 12 \\
\hline 0.45 & 0.52 & 0.995 & 5.2 & 0.33 & $23 \pm 3$ & 0.000 & 0.000 & 0.005 & 10 & 0 & 1 \\
\hline 0.45 & 0.61 & 0.986 & 4.8 & 0.39 & $24 \pm 4$ & 0.002 & 0.000 & 0.012 & 26 & 0 & 1 \\
\hline 0.45 & 0.75 & 0.982 & 4.3 & 0.40 & $26 \pm 4$ & 0.002 & 0.001 & 0.015 & 35 & 0 & 1 \\
\hline 0.45 & 0.90 & 0.490 & 9.2 & 0.13 & $25 \pm 4$ & 0.457 & 0.009 & 0.043 & 105 & 0 & 2 \\
\hline 0.45 & 1.00 & 0.469 & 7.9 & 0.15 & $25 \pm 4$ & 0.003 & 0.006 & 0.523 & 140 & 2 & 2 \\
\hline 0.45 & 1.10 & 0.442 & 9.6 & 0.11 & $23 \pm 4$ & 0.008 & 0.006 & 0.545 & 191 & 4 & 4 \\
\hline 0.45 & 1.25 & 0.416 & 9.2 & 0.06 & $20 \pm 3$ & 0.008 & 0.004 & 0.572 & 295 & 6 & 7 \\
\hline 0.60 & 0.52 & 0.997 & 4.5 & 0.40 & $21 \pm 3$ & 0.000 & 0.000 & 0.003 & 6 & 0 & 1 \\
\hline 0.60 & 0.61 & 0.989 & 4.2 & 0.38 & $24 \pm 3$ & 0.002 & 0.003 & 0.007 & 19 & 1 & 1 \\
\hline 0.60 & 0.75 & 0.512 & 8.1 & 0.09 & $20 \pm 4$ & 0.468 & 0.003 & 0.018 & 41 & 0 & 2 \\
\hline 0.60 & 0.90 & 0.499 & 8.1 & 0.13 & $18 \pm 3$ & 0.005 & 0.005 & 0.491 & 93 & 3 & 2 \\
\hline 0.60 & 1.00 & 0.460 & 12.1 & 0.11 & $14 \pm 2$ & 0.003 & 0.003 & 0.535 & 134 & 3 & 4 \\
\hline 0.60 & 1.10 & 0.454 & 10.9 & 0.05 & $11 \pm 2$ & 0.013 & 0.003 & 0.530 & 150 & 5 & 6 \\
\hline 0.75 & 0.52 & 0.998 & 4.1 & 0.26 & $23 \pm 4$ & 0.000 & 0.001 & 0.001 & 4 & 0 & 1 \\
\hline 0.75 & 0.61 & 0.996 & 4.9 & 0.39 & $23 \pm 3$ & 0.002 & 0.001 & 0.001 & 8 & 0 & 1 \\
\hline 0.75 & 0.75 & 0.493 & 10.1 & 0.12 & $9 \pm 2$ & 0.001 & 0.001 & 0.506 & 32 & 0 & 2 \\
\hline 0.75 & 0.90 & 0.484 & 10.0 & 0.14 & $7 \pm 2$ & 0.003 & 0.004 & 0.510 & 68 & 2 & 2 \\
\hline 0.75 & 1.00 & 0.470 & 20.0 & 0.13 & $6 \pm 2$ & 0.003 & 0.002 & 0.525 & 111 & 3 & 3 \\
\hline 0.90 & 0.52 & 0.999 & 4.3 & 0.45 & $16 \pm 3$ & 0.000 & 0.001 & 0.000 & 2 & 0 & 1 \\
\hline 0.90 & 0.61 & 0.504 & 10.0 & 0.14 & $8 \pm 2$ & 0.000 & 0.002 & 0.494 & 10 & 1 & 2 \\
\hline 0.90 & 0.75 & 0.496 & 13.9 & 0.16 & $4 \pm 1$ & 0.002 & 0.000 & 0.502 & 23 & 0 & 3 \\
\hline 0.90 & 0.90 & 0.492 & 14.5 & 0.12 & $3 \pm 1$ & 0.001 & 0.000 & 0.507 & 42 & 0 & 4 \\
\hline 1.00 & 0.52 & 0.502 & 9.6 & 0.12 & $6 \pm 2$ & 0.000 & 0.001 & 0.498 & 5 & 0 & 2 \\
\hline 1.00 & 0.61 & 0.502 & 13.8 & 0.11 & $5 \pm 1$ & 0.002 & 0.001 & 0.495 & 13 & 0 & 2 \\
\hline 1.00 & 0.75 & 0.499 & 16.6 & 0.20 & $3 \pm 1$ & 0.001 & 0.000 & 0.499 & 16 & 1 & 2 \\
\hline 1.10 & 0.52 & 0.501 & 12.6 & 0.09 & $4 \pm 1$ & 0.000 & 0.001 & 0.498 & 4 & 0 & 2 \\
\hline 1.10 & 0.61 & 0.499 & 17.2 & 0.12 & $2 \pm 1$ & 0.001 & 0.000 & 0.500 & 5 & 0 & 2 \\
\hline 1.25 & 0.52 & 0.501 & 48.4 & 0.07 & $1 \pm 1$ & 0.000 & 0.000 & 0.499 & 2 & 0 & 2 \\
\hline
\end{tabular}




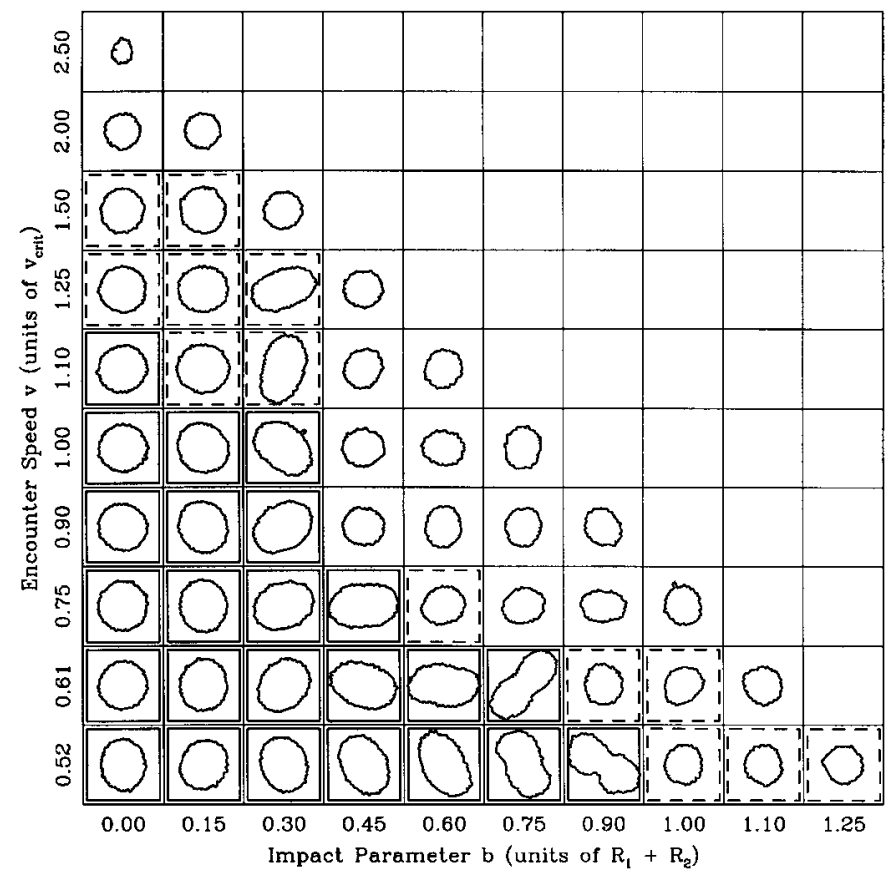

FIG . 2. Projected shape of the largest remnant at the end of each Model A run as a function of $b$ and $v$. At this scale each grid square measures $4 \mathrm{~km}$ on a side. Solid inner squares indicate remnants that retain at least $90 \%$ of the system mass; dashed squares indicate remnants with at least $50 \%$. Critical dispersal generally corresponds to the transition from solid to dashed, although in some cases a sizeable fragment may be about to accrete with the remnant. Table I gives additional data for this model.

more mass is carried away from the system, generally resulting in smaller $P$ values. As $b$ increases, so does the net angular momentum, resulting in faster spins (smaller $P$ ). This trend continues until $b \sim 1$ which corresponds to a grazing collision. In this case, the encounter generally does not result in a merger so the "remnant" is effectively one of the initial bodies plus or minus some mass exchange. Mass exchange and/or tidal torquing following deformation converts orbital angular momentum into spin angular momentum. As $b$ increases further, there is little spin-up, since torquing becomes less effective. All of these trends can be seen in the table.

Similarly, $\varepsilon$ depends on the total angular momentum of the system. Larger angular momentum allows the remnant to support a more elongated shape as long as most of the system mass ends up in the remnant ( $\sim 75 \%$, from the table). Consequently, there is also a relationship between $\varepsilon$ and $P$ : smaller $P$ values correspond to larger $\varepsilon$ values, in general. The smallest $P$ in the table is $4.1 \mathrm{~h}$ with $\varepsilon=0.26$; the largest $\varepsilon$ is 0.45 with $P=4.3 \mathrm{~h}$. These values are within the classical limit for mass retention at the surface,

$$
P_{\text {crit }} \simeq \frac{1}{1-\varepsilon} \sqrt{\frac{3 \pi}{G \rho}},
$$

where $\rho$ is the bulk density and we have assumed $a_{2}=a_{3}$. In this expression $P_{\text {crit }}=2.3 \mathrm{~h}$ for a spherical rubble pile with $\rho=2 \mathrm{~g} \mathrm{~cm}^{-3}$ and increases to infinity as $\varepsilon \rightarrow 1$.

The sixth column in Table I, labeled $f_{\text {mix }}$, gives the mean percentage mixing fraction and standard deviation after 100 repeated measurements (recall that the mixing calculation subvolumes are chosen radomly-cf. Section 2.7.3). The errors are a small fraction of the mean except when the mixing fraction itself is small. In the head-on case, $f_{\text {mix }}$ shows a simple trend of generally increasing with impact energy with a dip at the highest energy probably due to increased statistical fluctuation (the remnants are smaller). For most $b$ values the situation is more complicated depending on whether the impactors accrete into a single body or exchange mass while remaining two separate bodies. For the largest $b$, little mass is exchanged, so the bodies remain essentially unmixed.

The next three columns give dynamical information about the remaining mass of the system, i.e., the material not incorporated in the largest remnant. Generally most of this mass is escaping from the largest remnant $\left(M_{\mathrm{esc}}\right)$. Typically only small amounts $(<10 \%)$ of mass are accreting $\left(M_{\mathrm{acc}}\right)$ and/or orbiting $\left(M_{\mathrm{orb}}\right)$. In two cases, however, $M_{\text {acc }}$ is close to $50 \%$; these are instances of near escape that were too computationally expensive to run until final accretion and represent the transition from a high- to medium-mass remnant.

The final three columns contain information about the particle groupings at the end of each run. The number of free particles $\left(n_{1}\right)$ increases dramatically with $v$, but decreases with $b$. This trend is also seen in the number of two-particle groups $\left(n_{2}\right)$ and discrete rubble piles $(n)$. Groups can form either from accretion among the free particles due to gravitational instability or from being stripped off as a clump during the collision event. Note that $n$ is always at least 1 because the remnant is included.

In summary, the outcomes of this model depend in a natural way on the total angular momentum and impact energy of the system (both related to $b$ and $v$ ). Larger $b$ results in more elongated remnants with higher spins and reduced mixing. Larger $v$ results in greater mass loss and increased mixing. In the remaining sections we explore how these trends are modified for nonidentical or spinning bodies.

3.1.2. Model B: Equal size, spin. In Model B we added a spin component to the impactors. The spin vectors are oriented perpendicular to the orbital plane (i.e., along the $\pm z$ axis). The rotation period of the impactors is $6 \mathrm{~h}$, the median rotation period of near-Earth asteroids (Bottke et al. 1997). We investigated three cases: in Model B1 the spins of the impactors have opposite orientation; in Model B2 and B3 the spins have the same orientation but the impactors have opposite $y$ offsets (Fig. 3). By symmetry, these cases test all the unique $z$ angular momentum combinations (spin + orbital). The remaining parameters are identical to those in Model A.

Figures $4 \mathrm{a}-4 \mathrm{c}$ summarize the results for Models B1, B2, and B3, respectively (Fig. 1d is a snapshot sequence of a Model B1 run). The general trends seen in Fig. 4 are similar to those seen in Fig. 2. The head-on cases tend to result in spherical 


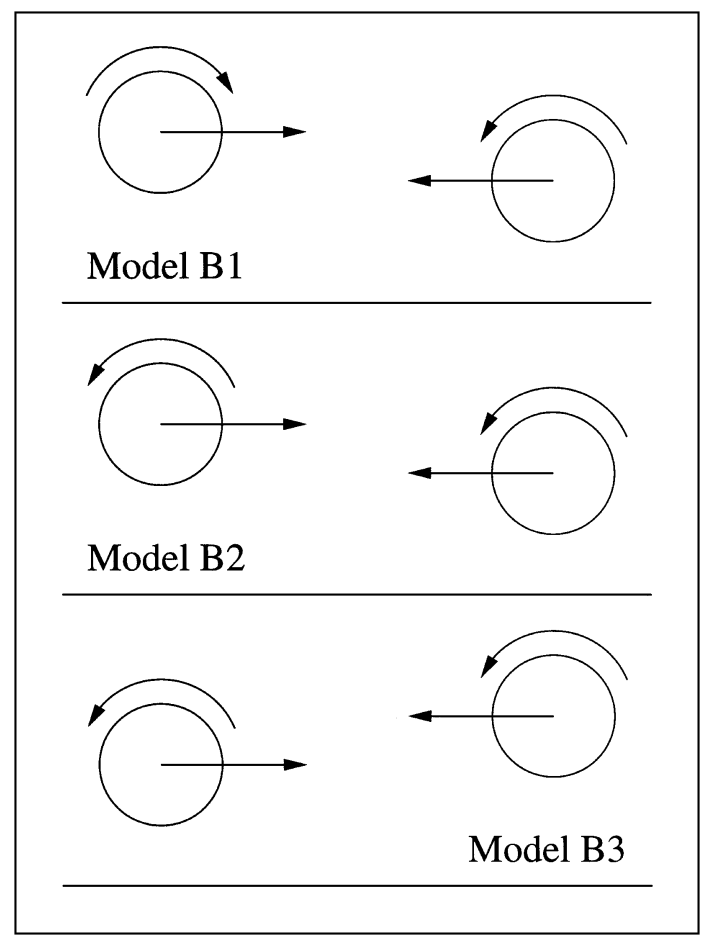

FIG. 3. Illustration of the spin sense for the Model B impactors. In Model B1, the impactors have opposite spin; in B2 they have the same spin, oppositely aligned with the orbital angular momentum; in B3 the spins are aligned with the orbital angular momentum.

remnants of decreasing mass with increasing $v$. The elongation of the remnants tends to increase with an increase in $b$, up to a point. Of the three models note that Model B1 is the most similar to Model A. This is because Model B1 has the same amount of net angular momentum in the system since the spin components of the impactors cancel. Model B2 and Model B3, however, have smaller and larger net angular momentum in the system, respectively, than Model A or B1. This is reflected in the number of runs with fast-rotating and/or elongated remnants (Table II). Models A and B1 have an intermediate number of runs with extreme $P$ and/or $\varepsilon$ values compared with Model B2 or B3 (Model $\mathrm{C}$ is a special case discussed in the next section).

Model B1 does differ from Model A in one important respect. As seen in Fig. $4 \mathrm{a}$, some of the remnants in this model (e.g., $b=0.30, v=1.10 ; b=0.60, v=0.61$ ) have unique asym-

\section{TABLE II}

\section{C omparison of R uns with Extreme $P$ and $e$ Values (Section 3.1.2)}

\begin{tabular}{lcc}
\hline Model & No. with $P \leq 5 \mathrm{~h}$ & No. with $e \geq 0.35$ \\
\hline A & 11 & 8 \\
B1 & 10 & 8 \\
B2 & 10 & 2 \\
B3 & 15 & 10 \\
C & 0 & 0 \\
\hline
\end{tabular}

metries (i.e., broken eightfold symmetry). This is because before the encounter one of the bodies is spinning prograde while the other body is spinning retrograde with respect to the orbit. The prograde rotator has larger angular momentum with respect to the center of mass of the system than its retrograde counterpart, consequently, it suffers more mass loss. This is analogous to the resistance of retrograde rotators to tidal disruption (Richardson et al. 1998).

To summarize other quantitative results, $24 \%$ of the Model B1 runs resulted in net erosion, while this value was $29 \%$ for B2, and $40 \%$ for B3. The mixing statistics are generally similar to those for Model A, namely that larger disruption resulted in more mixing. As for ejecta statistics, again no more than about $2 \%$ by mass remains in orbit around the remnant in all cases, while a somewhat larger percentage is destined to reaccrete (no more than $\sim 6 \%$, except for a few cases where components of a future contact binary were on slow-return trajectories). The distribution of fragments $\left(n_{1}, n_{2}\right.$, and $\left.n\right)$ followed trends similar to those of Model A.

3.1.3. Model C: Unequal size, no spin. In Model C we used two different-sized impactors with no initial spin: one large sphere of 1357 particles and $1 \mathrm{~km}$ radius, and one small sphere of 717 particles and $0.46 \mathrm{~km}$ radius, keeping the bulk densities the same $\left(2 \mathrm{~g} \mathrm{~cm}^{-3}\right)$ and the total number of particles similar to the previous models. Hence the larger sphere is 10 times the mass of the smaller sphere and the particles in the two impactors are different sizes (the smaller body has smaller particles, to ensure adequate resolution). We caution that the difference in particle sizes implies different packing efficiencies (porosities) which may affect the outcome (cf. Section 3.3). Both impactors were equilibrated using the same process as before. Note that the parameter space investigated is different from the previous models, primarily for better sampling of the tidal regime (large $b$, small $v$ ). For this model, $v_{\text {crit }}=2.9 \mathrm{~m} \mathrm{~s}^{-1}$. As for the previous models, the minimum $v$ is twice the value corresponding to $v_{\infty}=0$.

In Fig. 4d it is evident that most collisions result in net growth of the larger body (only 9 cases, or $16 \%$, result in net erosion, e.g., remnants with less than $90 \%$ of the total mass of the system). None of the encounters resulted in critical dispersal and only the highest-speed cases resulted in violent disruption of the combined system (i.e., $b=0, v=2.5$ ). Also the remnants are all roughly spherical (the largest $\varepsilon=0.26$ ). Figure $1(\mathrm{e})$ shows a typical encounter: the small body is pulverized and in this case planes off a chunk of the larger body (so $n_{1}$ is typically a few hundred in all runs except the most grazing, while $n_{2}$ and $n$ remain small, $\lesssim 10)$. Most of the smaller fragments escape the system, a tiny fraction $(<1 \%$ by mass) go into orbit around the remnant, while the remaining fragments return and blanket the remnant in the equatorial plane. The largest concentration of smaller particles is at the impact site. The rotation periods of the remnants in this model are typically long compared to those of the previous models due to the larger rotational inertia of the bigger impactor. Finally, there was little tidal interaction seen in any of the cases, suggesting even the minimum $v$ was 

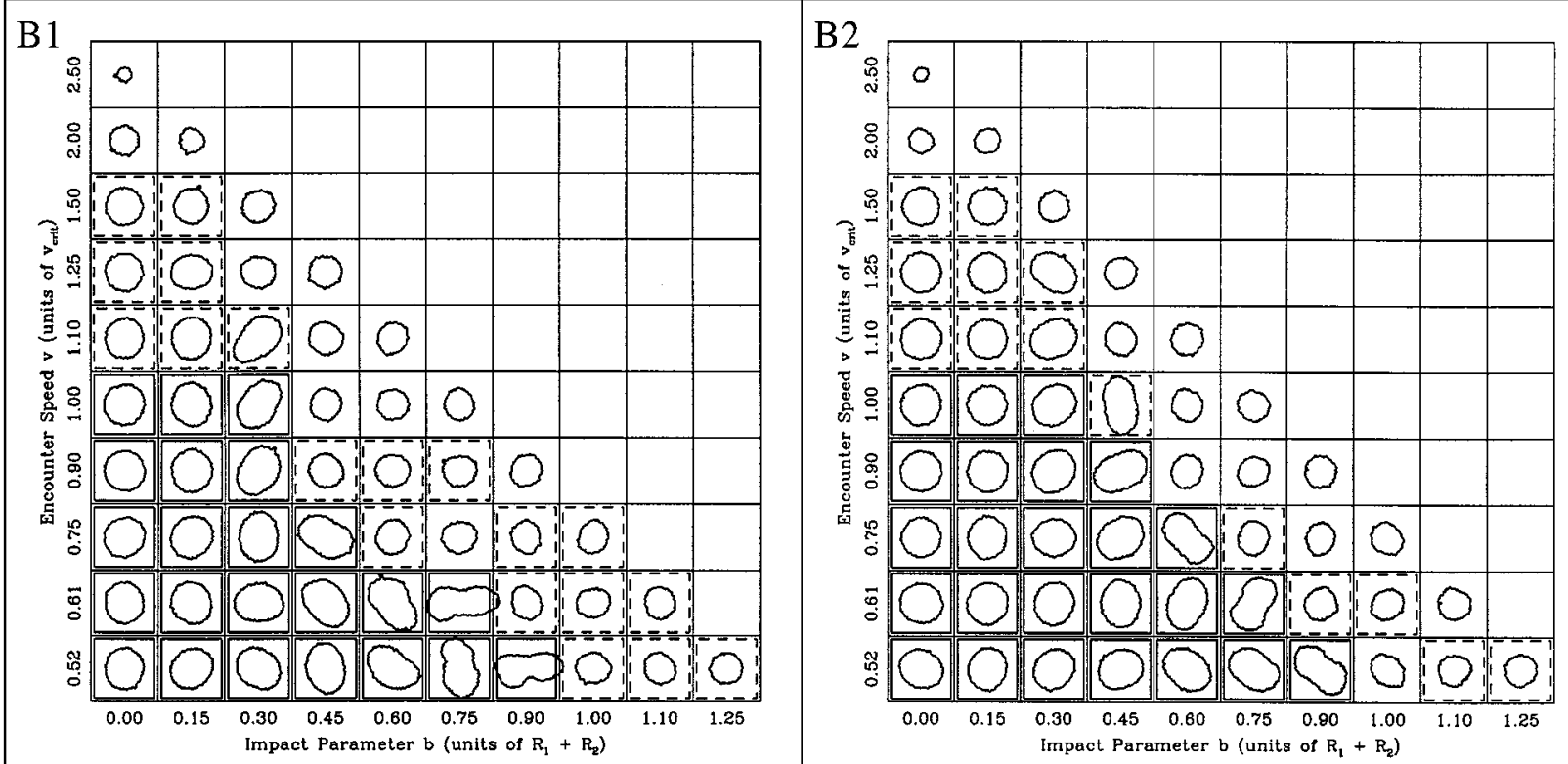

B3
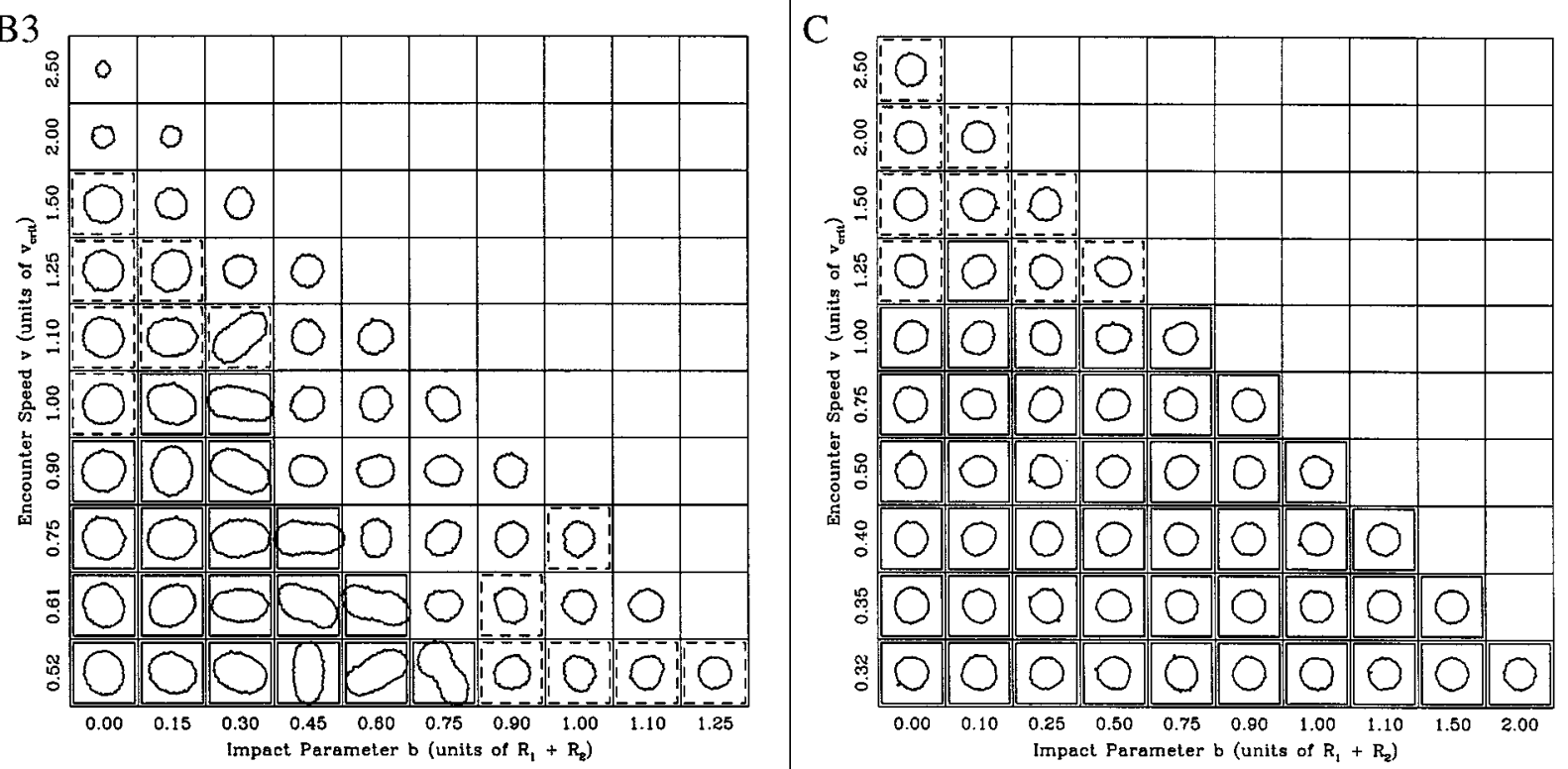

FIG . 4. Remnant shapes for the remaining parameter space models. The model is indicated in the top left of each plot. Compare with Fig. 2.

too large. Unfortunately, smaller $v$ would result in stronger path deflections, making interpretation more difficult.

\subsection{Coefficient of Restitution Test}

The energy change in the center-of-mass frame of a system of two smooth, colliding spheres is given by (e.g., Araki and Tremaine 1986)

$$
\Delta E=-\frac{1}{2} \mu\left(1-\epsilon_{\mathrm{n}}^{2}\right) v_{\mathrm{n}}^{2},
$$

where $v_{n}$ is the component of relative velocity normal to the mutual surfaces at the point of contact, and $\mu$ and $\epsilon_{\mathrm{n}}$ have the usual definitions. Hence, as $\epsilon_{\mathrm{n}} \rightarrow 0$, all the impact energy-less a geometric factor that depends on $b$-is dissipated. Although a collision between two rubble piles consists of many individual particle collisions, the dependence of $\Delta E$ on $\epsilon_{\mathrm{n}}$ suggests that $Q_{\mathrm{D}}^{*}$ will depend on $\epsilon_{\mathrm{n}}$ in a similar way, namely that smaller $\epsilon_{\mathrm{n}}$ implies larger $Q_{\mathrm{D}}^{*}$.

A simple test bears this out. Table III and Fig. 5 summarize the effect of varying $\epsilon_{\mathrm{n}}$ for one of the Model $A$ runs $(b=0.15, v=$ 2.00; cf. Fig. 1(b)). The general trend is clear: as $\epsilon_{\mathrm{n}}$ decreases, the size of the largest remnant increases (note the large $M_{\text {acc }}$ value for the $\epsilon_{\mathrm{n}}=0.2$ case, indicating that a big fragment is about to merge with the remnant, giving it the largest mass of 
TABLE III

Effect of Varying D issipation in Model A R un $b=0.15$, $v=2.00$ (Section 3.2)

\begin{tabular}{rrrrrrrr}
\hline$\epsilon_{\mathrm{n}}$ & $M_{\text {rem }}$ & $M_{\text {acc }}$ & $M_{\text {orb }}$ & $M_{\text {esc }}$ & $n_{1}$ & $n_{2}$ & $n$ \\
\hline 0.2 & 0.196 & 0.177 & 0.028 & 0.598 & 420 & 29 & 44 \\
0.5 & 0.364 & 0.006 & 0.004 & 0.626 & 483 & 31 & 33 \\
0.6 & 0.301 & 0.007 & 0.007 & 0.685 & 597 & 31 & 41 \\
0.7 & 0.284 & 0.029 & 0.006 & 0.682 & 746 & 32 & 39 \\
0.8 & 0.275 & 0.009 & 0.005 & 0.710 & 939 & 24 & 30 \\
0.9 & 0.040 & 0.006 & 0.005 & 0.949 & 1484 & 63 & 26 \\
1.0 & 0.001 & 0.000 & 0.000 & 0.999 & 1904 & 3 & 0 \\
\hline
\end{tabular}

all the runs). Runs with smaller $\epsilon_{\mathrm{n}}$ form discrete rubble piles out of the collision debris faster and more efficiently than those with larger $\epsilon_{\mathrm{n}}$. For $\epsilon_{\mathrm{n}}=1$, no rubble piles actually form. The strong dependence on $\epsilon_{\mathrm{n}}$ suggests that further study is needed to determine the value most representative of true rubble pile collisions.

\subsection{High-Resolution Models}

In order to test the degree to which particle resolution affects the collision outcome, we performed two high-resolution runs using parameters drawn from Model A. Each impactor for this test consisted of 4995 identical particles, more than 5 times the number used in the parameter space runs (Section 3.1). The progenitor needed $1 \mathrm{CPU}$ day to equilibrate using 2 processors on the SGI Origin 200. At equilibrium, the code was performing $\sim 4 \times 10^{4}$ collisions per step, with each step requiring $\sim 140 \mathrm{~s}$ wallclock time. The enhanced packing efficiency of the highresolution impactors plus their randomized orientations makes detailed comparison with the low-resolution runs difficult. However, we would expect the general trends to be similar (i.e., outcome class, etc.). Figure 6a shows snapshots shortly after the initial impact comparing the low- and high-resolution Model A runs with $b=0.30, v=1.25$. Figure $6 \mathrm{~b}$ shows post-reaccretion snapshots for $b=0.60, v=0.61$. These runs were chosen because they are moderately well separated in $b-v$ space while still being representative of the complex intermediate-energy regime (cf. Fig. 2). The expense of these calculations precluded a more thorough sampling.

Both high-resolution runs in this test show evolution similar to that of their low-resolution counterparts. In Fig. 6a, the impactors mutually penetrate and lose most of their relative orbital energy (the bodies will eventually accrete into a single massive remnant). Note the presence of the "mass bridge" between the two bodies in both cases. The rotational phase and penetration
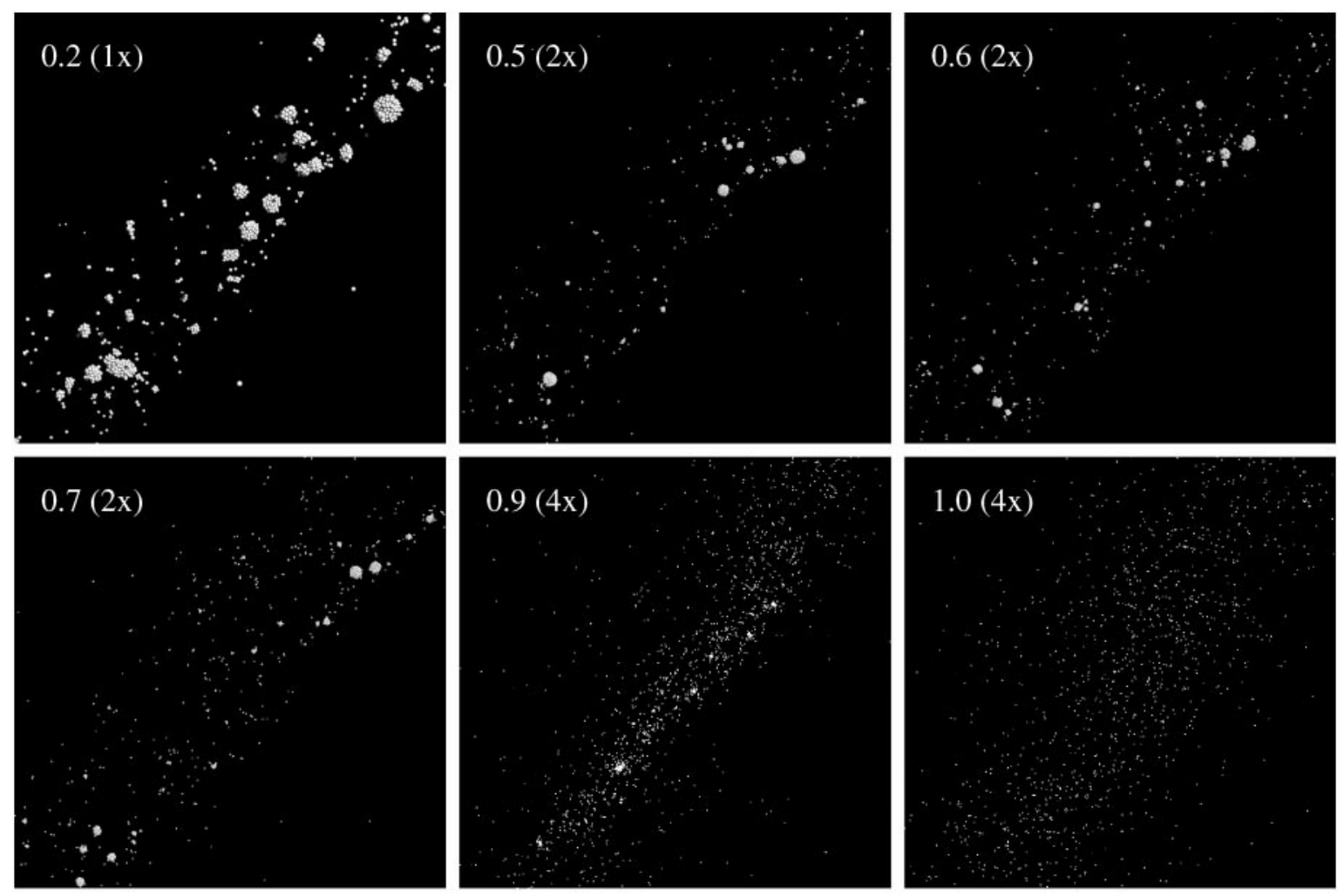

FIG . 5. Snapshots showing the effect of varying $\epsilon_{\mathrm{n}}$ for the Model A run with $b=0.15, v=2.00$ (cf. Fig. $1 \mathrm{~b}$ ). Each snapshot was taken about $6.5 \mathrm{~h}$ after impact. The chosen $\epsilon_{\mathrm{n}}$ value and camera zoom-out factor are shown in the top left of each frame. For clarity, no color or shading distinction is made between the particles of the original impactors, and the $\epsilon_{\mathrm{n}}=0.9$ and $\epsilon_{\mathrm{n}}=1.0$ frames have been brightened. From these snapshots and the statistics in Table III it can be seen that rubble pile formation favors smaller $\epsilon_{\mathrm{n}}$ values. The differences at the extremes are dramatic. 


\section{Low Resolution}
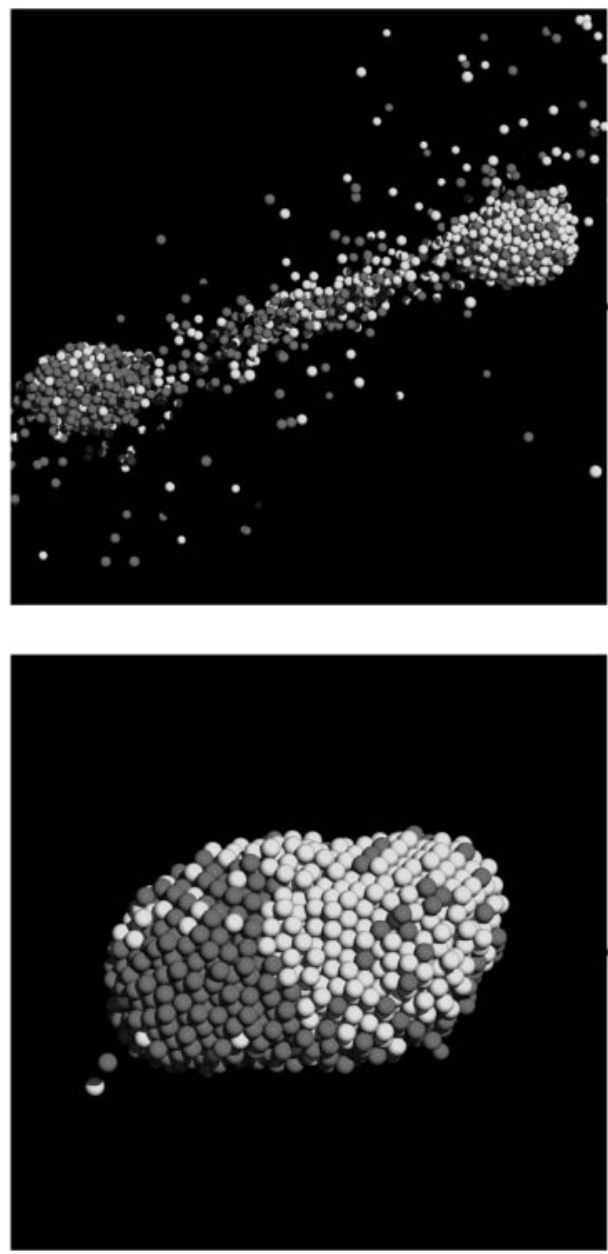

High Resolution
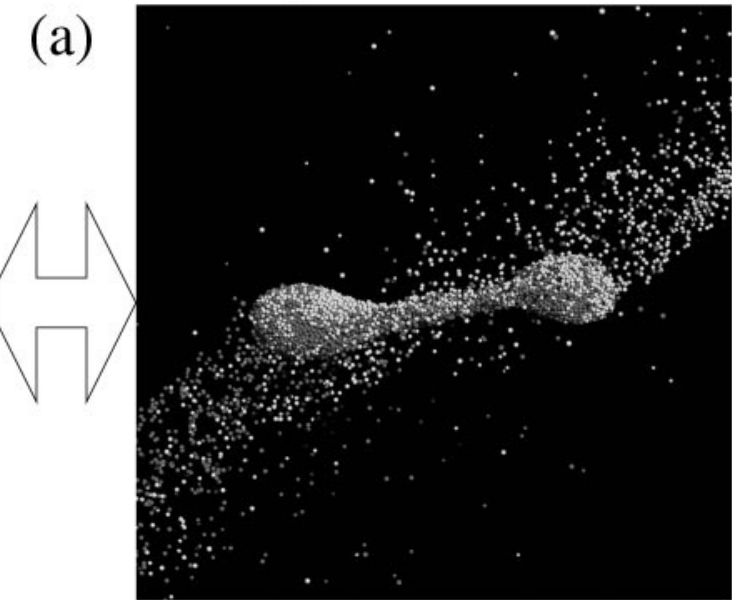

(b)

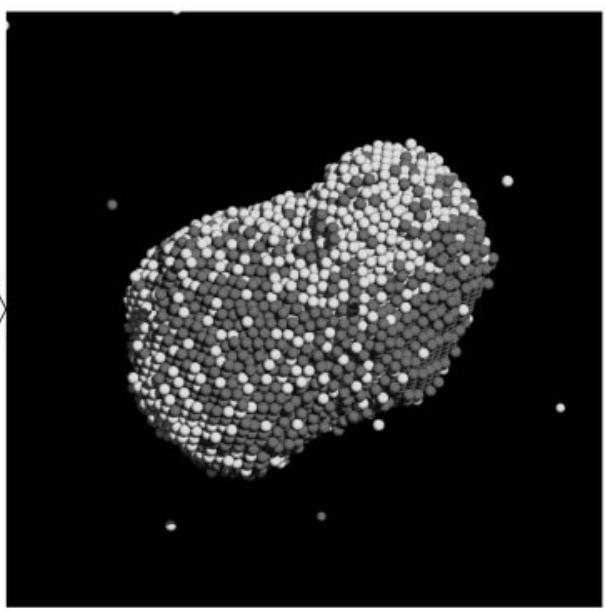

FIG . 6. Comparison of two Model A runs performed at low resolution ( 955 particles per rubble pile; left column) and high resolution (4995 particles per rubble pile; right column). The run parameters are: (a) $b=0.30, v=1.25$; (b) $b=0.60, v=0.61$. The evolution is similar in both cases, with differences attributable to packing efficiency, initial orientation, and possibly enhanced dissipation at higher resolution.

distance differ somewhat, perhaps indicating that higher resolution (and hence lower porosity) gives rise to more efficient dissipation, by increasing the degrees of freedom. In Fig. 6b, the final shape of the reaccreted body at low and high resolution is similar, but there is more structural detail in the high-resolution remnant, e.g., the depression on the upper surface. The remnant mass and rotation period are comparable at this instant: 0.985 and $4.2 \mathrm{~h}$, respectively, at low resolution; 0.987 and $4.1 \mathrm{~h}$ at high resolution. We conclude that higher resolution may give insight into the more detailed aspects of reaccumulation, but low resolution is sufficient for a broad sampling of parameter space.

\section{DISCUSSION}

\subsection{Critical Dispersal Threshold}

Despite the large number of runs carried out for this investigation, the data are still too sparse in each model to reliably derive a generalized expression for the retained mass (remnant plus accreting and orbiting material) as a function of $b$ and $v$; i.e., $1-M_{\text {esc }}=f(b, v)$. However, we can solve for the critical contour $f(b, v)=0.5$, which is well sampled by our choice of parameter space (for Model C, we solve for $f(b, v)=0.9$, the point of net erosion for the larger impactor). Our method is to perform bilinear interpolation of our $b$-versus- $v$ results onto a regular grid, root solve using Newton's method for the $v$ value that gives a remnant mass of 0.5 at each grid line in $b$ (we chose 20 lines for smooth sampling), and fit the resulting values to a functional form. After some experimentation, we found that the contour is best represented by a Gaussian:

$$
\left.v\right|_{f=0.5} \equiv v_{*}=\alpha \exp \left[-\frac{(b-\beta)^{2}}{\gamma}\right]+\delta ;
$$

where $\alpha, \beta, \gamma$, and $\delta$ are parameters to be determined by nonlinear least-squares fitting. 


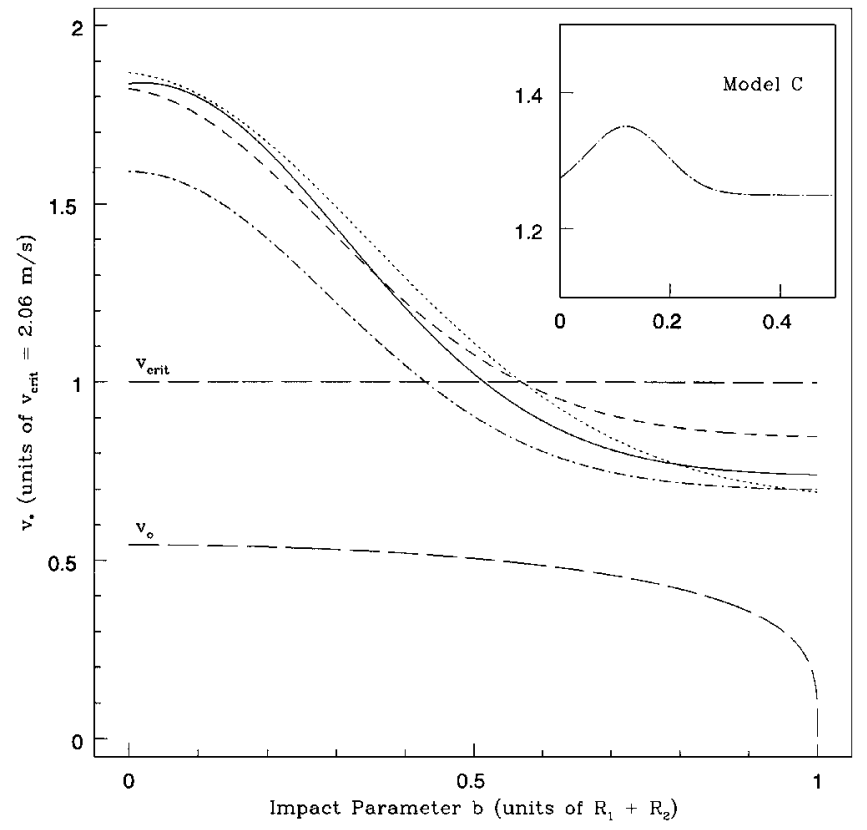

FIG. 7. Best fits to Eq. (9) for $M_{\text {rem }}=0.5$ contours, $b \leq 1$ : Model A (solid line; $\alpha=1.10 \pm 0.03, \beta=0.02 \pm 0.01, \gamma=0.17 \pm 0.01, \delta=0.74 \pm 0.02), \mathrm{B} 1$ (short dashed; $\alpha=0.98 \pm 0.01, \beta=-0.024 \pm 0.005, \gamma=0.190 \pm 0.005, \delta=$ $0.846 \pm 0.006$ ), B2 (dotted; $\alpha=1.2 \pm 0.1, \beta=-0.02 \pm 0.04, \gamma=0.27 \pm 0.05$, $\delta=0.67 \pm 0.06$ ), B3 (dot-short dashed $\alpha=0.89 \pm 0.03, \beta=0.00 \pm 0.02, \gamma=$ $0.17 \pm 0.02, \delta=0.70 \pm 0.02$ ), and $\mathrm{C}$ (inset; $\alpha=0.10 \pm 0.03, \beta=0.12 \pm 0.04$, $\gamma=0.010 \pm 0.009, \delta=1.25 \pm 0.02$, for the $M_{\mathrm{rem}}=0.9$ contour, $\left.b \leq 0.5\right)$. The $v_{\mathrm{o}}$ curve is the $v_{\infty}=0$ contour. The value of $v_{\text {crit }}$ is given by Eq. (1).

Figure 7 gives the best-fit values of the Gaussian parameters along with their $1-\sigma$ uncertainties for each of the parameter space models. Note that the fits are marginally consistent with $\beta=0$, i.e., no $b$ offset, except for Model C. The differences between the fits (except Model C) are slight, but they follow the trend mentioned in Section 3.1.2, namely that Model B2 has a higher disruption threshold than Model B3, with Models A and $\mathrm{B} 1$ having intermediate thresholds. Model $\mathrm{C}$ has a broader distribution that is somewhat offset in $b$, but inspection of the other models shows similar trends for the 0.9 contour.

\subsection{Debris Size Distributions}

Combining all 275 runs of Models A, B, and C, we find the largest primary (remnant) mass is 0.999 (so there were no perfect mergers), the largest secondary mass is 0.498 (there was always some grazing mass exchange, at least in Models A and B), and the largest tertiary mass is 0.073 . The smallest primary mass is 0.038 . In Models A and B, the most common outcome was an even split in mass between the primary and secondary, since most runs at moderate to large $b$ resulted in little to no mass exchange between the impactors. For $b \leq 0.30\left(\phi \leq 17^{\circ}\right)$, the normalized primary mass function is well approximated by a curve of the form $n(m) \propto 1 /\left(1-m^{2}\right), m<1$ (Fig. 8).

\subsection{Debris Spatial Distributions}

In cases where debris escapes the central remnant, the ejected material is invariably concentrated in a plane normal to the orbital $(z=0)$ plane, although in some cases material can be spread out in the orbital plane as two returning fragments coalesce. For our head-on collisions $(b=0)$, the dispersal plane is normal to the $x$ axis. For $b>0$, the plane is initially normal to the impact angle $\phi$, but rotational inertia from the orbital motion causes the dispersal plane to overshoot this value. As usual, initial spin may help or hinder this process (note for Model B3, $\phi \sim-\sin ^{-1} b$ ).

Figure 9 illustrates the anisotropic distribution of ejecta as projected to the orbital plane for four of the five runs shown in Fig. 1. In the equal-mass cases the angular distribution is bi-modal, with the peaks roughly $180^{\circ}$ apart. For the plot representing Fig. 1b, the peaks are of unequal amplitude since the remnant is relatively small and displaced from the system center of mass. The one unequal-mass case is unimodal, indicating that debris was scattered preferentially in one direction (roughly $30^{\circ}$ measured counterclockwise from the $x$ axis), as seen in Fig. 1e.

Generally, the $z$ distributions are sharply peaked near the largest remnant but some particles end up many hundreds of kilometers away. Recall that the tidal field of the Sun is not included in our simulations. If it were, these particles would be well outside the remnant's Hill sphere at $1 \mathrm{AU}$ (for example),

$$
r_{\mathrm{H}}=(210 \mathrm{~km})\left[\frac{a}{1 \mathrm{AU}}\right]\left[\frac{R}{1 \mathrm{~km}}\right]\left[\frac{\rho}{2 \mathrm{~g} \mathrm{~cm}^{-3}}\right]^{1 / 3},
$$

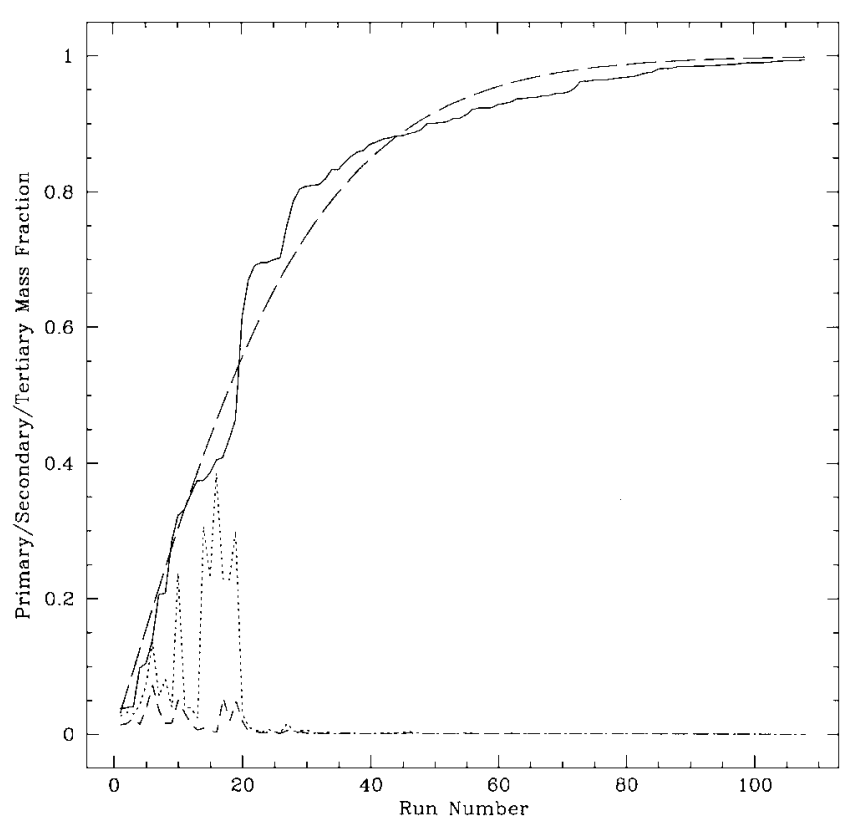

FIG . 8. Primary (solid line), secondary (dotted), and tertiary (short dashed) mass fractions of every Model A and B run with $b \leq 0.30$, sorted by primary mass. The long-dashed line was obtained by integrating a rough fit to the primary mass function of the form $1 /\left(1-m^{2}\right)$. 


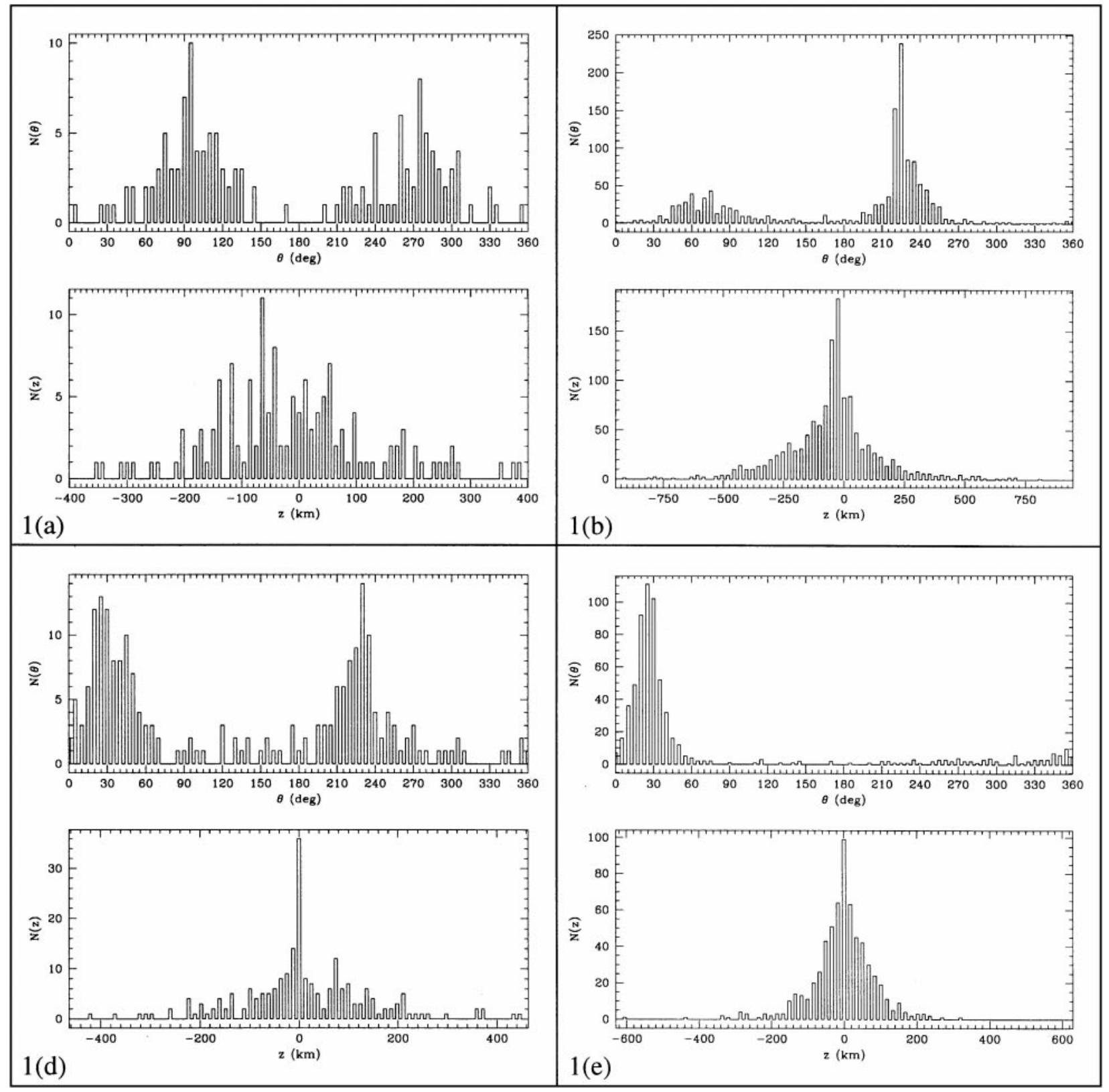

FIG . 9. Debris dispersal patterns in the initial orbital plane relative to the largest remnant for the runs labeled (a), (b), (d), and (e) in Fig. 1. The $\theta$ histograms are binned in $5^{\circ}$ increments. Only particles with projected distances in the $z$ plane exceeding twice the remnant radius were included.

where $a$ is the distance to the Sun, $R$ is the radius of the remnant, and $\rho$ is its bulk density. Regardless, most of these particles would escape the remnant, even without the solar tides.

\subsection{Outcome Probability}

For a given impact parameter and speed distribution, the probability of a net accretional (as opposed to net erosional) outcome can be estimated from Eq. (9). Suppose we set $b=0.7$, which corresponds to $\phi=45^{\circ}$, the most probable impact angle for randomly flying projectiles striking a spherical target (Love and
Ahrens 1996). A monodispersive population of bodies with a Maxwellian distribution of speeds whose rms equals the escape speed $v_{\mathrm{e}}$ from a particle's surface has the following normalized distribution function in relative speed (e.g., Binney and Tremaine 1987, Problem 7-3):

$$
g(v) \mathrm{d} v=\frac{1}{2 \sqrt{\pi} v_{\mathrm{e}}^{3}} \exp \left(-\frac{v^{2}}{4 v_{\mathrm{e}}^{2}}\right) v^{2} \mathrm{~d} v
$$

The probability of a net accretional impact for hyperbolic 
encounters with $b=0.7$ is then

$$
P[f(b=0.7, v) \geq 0.5]=\frac{\int_{v_{\mathrm{o}}}^{v_{*}} g(v) \mathrm{d} v}{\int_{v_{\mathrm{o}}}^{\infty} g(v) \mathrm{d} v},
$$

where $v_{\mathrm{o}}$ is the initial speed corresponding to $v_{\infty}=0$ from Eq. (3) and $v_{*}$ is obtained from Eq. (9). For Model A, we have $v_{\mathrm{e}}=0.51$, $v_{\mathrm{o}}=0.22$, and $v_{*}=0.81$. Solving Eq. (12) numerically we find that the probability of an accretional impact in this case is $26 \%$. The probability of erosion is $1-P(f \geq 0.5)=74 \%$.

The full accretional cross section is obtained by integrating Eq. (12) over all impact parameters. The net accretion probability is then the ratio of this value to the geometrical cross section,

$$
P[f(b, v) \geq 0.5]=\frac{1}{\pi \int_{0}^{1} b \mathrm{~d} b} \pi \int_{0}^{1} b \mathrm{~d} b \frac{\int_{v_{\mathrm{o}}}^{v_{*}(b)} g(v) \mathrm{d} v}{\int_{v_{\mathrm{o}}(b)}^{\infty} g(v) \mathrm{d} v}
$$

where the dependence of $v_{\mathrm{o}}$ and $v_{*}$ on $b$ has been made explicit. Solving this equation we find the accretion probability increases to $35 \%$ only, since head-on collisions are relatively rare. Such a low value implies that this population of rubble piles may be hindered from forming planets. If rubble piles were common during the early stages of planet formation then perhaps collisions were more dissipative than modeled here. Alternatively, the accretion probability may be enhanced when there is a distribution of masses, a possibility that can only be tested with more simulations using impactors of varying size.

\subsection{Comparison with Previous Work (Gravity Regime)}

Using the fits to Eq. (9) we can estimate the value of $Q_{\mathrm{D}}^{*}$ (recall this is for $\epsilon_{\mathrm{n}}=0.8$; further runs are needed to determine the dependence on dissipation). Restricting ourselves to Model A with $b=0$, we find $Q_{\mathrm{D}}^{*} \sim 1.9 \mathrm{~J} \mathrm{~kg}^{-1}$. This lies very close to the Holsapple (1994), Durda et al. (1998), and gravitational binding energy curves described in Love and Ahrens (1996; see in particular their Eq. (2) and Fig. 7). It lies well off the extrapolation of their SPH results. In their paper they suggest that the discrepancy between their results and analytic or experimental results may arise from: (1) the local rather than global deposition of impact energy at the surface of the target; (2) the difference between the role of gravity in self-compression and ejecta retention; and (3) the finite size of the projectile. The present work, however, is similar to Love and Ahrens (1996) in all these respects, which suggests that the difference may be attributable to the adopted equation of state (an incompressible fluid in our case, compared with the Tillotson equation of state for granite in theirs) or a possible reolution problem in their simulations. Note that the SPH curve plotted in Fig. 7 of Love and Ahrens (1996) was for an impact angle of $\phi=45^{\circ}$, but this would amount to less than an order of magnitude difference in $Q_{\mathrm{D}}^{*}$.

If the outcome truly depends solely on the gravitational binding energy (ignoring the effect of dissipation for now as this re- quires further study), then we would expect $Q_{\mathrm{D}}^{*} \propto M / R \propto R^{2} \propto$ $M^{2 / 3}$. From our Model A point we can estimate the constant of proportionality: $Q_{\mathrm{D}}^{*} \sim 1.2 \times 10^{-6} R^{2} \sim 2.9 \times 10^{-9} M^{2 / 3}$. Further models with different $M$ are needed to confirm this result (our Model C case failed to sample the critical dispersal regime, so we cannot use it here).

Watanabe and Miyama (1992) found $M_{\mathrm{esc}} \propto v^{3}$ for their lowspeed, head-on SPH models (see Eq. (3.5.1) in their paper). We find a similar trend. For the $b=0$ outcomes of Model A, a leastsquares fit to the form

$$
M_{\mathrm{esc}}=\alpha v^{\beta}
$$

yields $\alpha=0.06 \pm 0.02$ and $\beta=3.2 \pm 0.1$. Evidently this relation must break down at large $v$, otherwise $M_{\text {esc }}$ would exceed unity. Indeed our only significant outlier is for our highest $v$ value (2.50), with $M_{\text {esc }}$ in this case $\sim 20 \%$ below the curve.

\section{CONCLUSIONS}

In summary, we have conducted a series of numerical simulations to create a partial map of the parameter space of rubble pile collisions at low impact speeds. The general trends can be summarized as follows: (1) larger impact angles result in more elongated, faster-spinning remnants; (2) larger impact speeds result in greater mass loss and increased mixing of the remnant; and (3) initial impactor spin can increase or reduce the rotation period and elongation of the remnant. It is also possible to create asymmetric shapes if the impactors have oppositely oriented spins. These general trends are directly related to the total energy and angular momentum of the system. In cases where one impactor is significantly larger than the other (Model C), the smaller body generally disrupts completely on impact, sometimes removing a modest fraction of the surface of the target body and sometimes redepositing material along the remnant's $z=0$ equator.

We have been able to generate a wide variety of remnant shapes, including spheroids, ellipsoids, contact binaries (peanut shaped and S shaped), and shapes with broken eightfold symmetry. It proved difficult to get a significant amount of material to orbit the remnant; most debris (98\%) either accreted onto the remnant or escaped from the system. We found no detached binaries of significant size, but $\sim 10 \%$ of the remnants in Models A and $\mathrm{B}$ are contact binaries. The coefficient of restitution appears to play a more important role in collisions than in tidal disruption and can strongly affect the number and size of post-impact rubble-pile fragments. Increased particle resolution (or reduced porosity) appears to augment dissipation and give rise to more complex shapes, but the effects are modest over a factor of 5 in particle number.

We found that the impact speed needed for critical dispersal is well represented by a Gaussian function of impact parameter. Given a velocity distribution it is possible to estimate the probability of either impactor gaining or losing mass as a result 
of the collision. At low impact angles with equal-size impactors the remnant mass function is roughly proportional to $1 /\left(1-m^{2}\right)$. Secondary and tertiary masses are typically finite but small, except for near-grazing encounters. Most material is ejected in a plane perpendicular to the axis of the initial motion and in some cases the debris can coalesce into smaller rubble piles.

We found $Q_{\mathrm{D}}^{*} \sim 2 \mathrm{~J} \mathrm{~kg}^{-1}$ for the head-on collisions in Model A and that $M_{\mathrm{esc}} \propto v^{3.2}$. The former result is in rough agreement with the theoretical gravity-regime model of Holsapple (1994). The latter relation agrees with Watanabe and Miyama (1992). We find that kilometer-sized rubble piles in general are much easier to disperse than previously thought. This may be due in part to our conservative choice for $\epsilon_{\mathrm{n}}(0.8)$. Although more work needs to be done, we believe that our simulations may provide a numerical basis for parameterizing collisions during the early stage of planet formation, when the planetesimals are dynamically cool and the dominant sizes are still $\lesssim 10 \mathrm{~km}$.

\subsection{Future Work}

In this study we were restricted to investigating the dependence of collision outcome primarily on impact parameter and impact speed. We also examined a few spin combinations, a model with unequal masses, and a single run with various values of the restitution coefficient. However, the parameter space is truly vast. Naturally we would like to test more values for the parameters we have already investigated, particularly the coefficient of restitution and the dependence on porosity. We also need a finer grid at small speed and near-grazing separation to fully investigate the tidal regime (this would also provide better data for comparison with stellar system collision models, e.g., Davies et al. 1991). However there are many other new parameters to explore. We would like to test the effect of changing the spin-axis orientations (beyond pure prograde or retrograde). We suspect this would result in even more unusual shapes. Nonspherical impactors with a variety of sizes would improve realism and provide better estimates of $Q_{\mathrm{D}}^{*}$. A spectrum of particle sizes could alter the effective dissipation as smaller particles fill the voids between larger ones. Adding surface friction could lead to steeper slopes and the possibility of simulating crater formation in large targets. We plan to add a simple model for compaction to allow higher impact speeds and compare with the porous models of Housen et al. (1999). We would like to track the movement of particles near the cores of our rubble piles and compare with the surface particles to study "scrambling" in a single rubble pile. There are so many possibilities that likely the only practical approach would be to randomly sample points in this vast parameter space to get a feel for the overall trends and then concentrate on the most interesting aspects in detail. We will carry out such work in the future.

\section{ACK NOW LED GMENTS}

We are indebted to E. Asphaug and D. Durda for extensive comments. We also thank W. Bottke, C. Dominik, K. Holsapple, G. Lake, C. Reschke, J. Stadel, B. Titus, and F. van den Bosch. This work was supported in part by the NASA
HPCC-ESS and Intel Technology 2000 Programs and a NASA Innovative Research grant. Ray-traced images were rendered using the Persistence of Vision Raytracer (POV-Ray version 3.02).

\section{REFERENCES}

Anderson, R. J. 1993. Computer science problems in astrophysical simulation. In Silver Jubilee Workshop on Computing and Intelligent Systems, pp. 48-61. Tata McGraw-Hill, New Delhi.

Anderson, R. J. 1996. Tree data structures for $N$-body simulation. 37th Symp. Found. Comput. Sci. 224-233.

Araki, S., and S. Tremaine 1986. The dynamics of dense particle disks. Icarus 65, 83-109.

Asphaug, E., and W. Benz 1996. Size, density, and structure of Comet Shoemaker-Levy 9 inferred from the physics of tidal breakup. Icarus 121, 225-248.

Asphaug, E., and H. J. Melosh 1993. The Stickney impact of Phobos: A dynamical model. Icarus 101, 144-164.

Asphaug, E., S. J. Ostro, R. S. Hudson, D. J. Scheeres, and W. Benz 1998. Disruption of kilometer-sized asteroids by energetic collisions. Nature 393, 437-440.

Beaugé, C., and S. J. Aarseth 1990. N-body simulations of planetary formation. Mon. Not. R. Astron. Soc. 245, 30-39.

Bentley, J. L., and J. H. Friedman 1979. Data structures for range searching. Comput. Surv. 11, 397-409.

Benz, W., and E. Asphaug 1999. Catastrophic disruptions revisited. Icarus 142, $5-20$.

Binney, J., and S. Tremaine 1987. Galactic Dynamics. Princeton Univ. Press, Princeton, NJ.

Bottke, W. F., and H. J. Melosh 1996a. The formation of asteroid satellites and doublet craters by planetary tidal forces. Nature 381, 51-53.

Bottke, W. F., and H. J. Melosh 1996b. The formation of binary asteroids and doublet craters. Icarus 124, 372-391.

Bottke, W. F., Jr., D. C. Richardson, and S. G. Love 1997. Can tidal disruption of asteroids make crater chains on the Earth and Moon? Icarus 126, 470-474.

Bottke, W. F., Jr., D. C. Richardson, P. Michel, and S. G. Love 1999. 1620 Geographos and 433 Eros: Shaped by planetary tides? Astron. J. 117, 19211928.

Davies, M. B., W. Benz, and J. G. Hills 1991. Stellar encounters involving red giants in globular cluster cores. Astrophys. J. 381, 449-461.

Durda, D. D. 1996. The formation of asteroidal satellites in catastrophic collisions. Icarus 120, 212-219.

Durda, D. D., R. Greenberg, and R. Jedicke 1998. Collisional models and scaling laws: A new interpretation of the shape of the main-belt asteroid size distribution. Icarus 135, 431-440.

Greenberg, R., J. Wacker, C. R. Chapman, and W. K. Hartman 1978. Planetesimals to planets: Numerical simulation of collisional evolution. Icarus 35, $1-26$.

Harris, A. W. 1996. The rotation rates of very small asteroids: Evidence for "rubble-pile" structure. Proc. Lunar Planet. Sci. Conf. 27th, 493-494.

Holsapple, K. A. 1994. Catastrophic disruptions and cratering of Solar System bodies: A review and new results. Planet. Space Sci. 42, 1067-1078.

Housen, K. R., K. A. Holsapple, and M. E. Voss 1999. Compaction as the origin of the unusual craters on the Asteroid Mathilde. Nature 402, 155-157.

Housen, K. R., R. M. Schmidt, and K. A. Holsapple 1991. Laboratory simulations of large scale fragmentation events. Icarus 94, 180-190.

Love, S. G., and T. J. Ahrens 1996. Catastrophic impacts on gravity dominated asteroids. Icarus 124, 141-155.

Love, S. G., F. Hörz, and D. E. Brownlee 1993. Target porosity effects on impact cratering, and collisional disruption. Icarus 105, 216-224. 
Merline, W. J., and 10 colleagues 1999. Discovery of asteroidal satellite S/1998 (45) 1. Bull. Am. Astron. Soc. 31, 1106.

Ostro, S. J., and 19 colleagues 1999. Radar and optical observations of Asteroid 1998 KY26. Science 285, 557-559.

Petit, J.-M., and M. Hénon 1987. A numerical simulation of planetary rings. I. Binary encounters. Astron. Astrophys. 173, 389-404.

Richardson, D. C. 1994. Tree code simulations of planetary rings. Mon. Not. R. Astron. Soc. 269, 493-511.

Richardson, D. C., E. Asphaug, and L. Benner 1995. Comet Shoemaker-Levy 9: A "rubble pile" model with dissipative collisions and gravitational perturbations. Bull. Am. Astron. Soc. 27, 1114.

Richardson, D. C., W. F. Bottke, Jr., and S. G. Love 1998. Tidal distortion and disruption of Earth-crossing asteroids (Paper I). Icarus 134, 47-76.

Richardson, D. C., T. Quinn, J. Stadel, and G. Lake 1999. Direct largescale $N$-body simulations of planetesimal dynamics (Paper II). Icarus 143, $45-59$.
Ryan, E. V., and H. J. Melosh 1998. Impact fragmentation: From the laboratory to asteroids. Icarus 133, 1-24.

Ryan, E. V., W. K. Hartmann, and D. R. Davis 1991. Impact experiments. 3. Catastrophic fragmentation of aggregate targets and relation to asteroids. Icarus 94, 283-298.

Veverka, J., and 16 colleagues 1997. Flyby of 253 Mathilde: Images of a C asteroid. Science 279, 2109-2114.

Wasson, J. T. 1985. Meteorites-Their Record of Early Solar System History. Freeman, New York.

Watanabe, S., and S. M. Miyama 1992. Collision and tidal interaction between planetesimals. Astrophys. J. 391, 318-335.

Wetherill, G. W., and G. R. Stewart 1993. Formation of planetary embryos: Effects of fragmentation, low relative velocity, and independent variation of eccentricity and inclination. Icarus 106, 190-204.

Yeomans, D. K., and 12 colleagues 1997. Estimating the mass of Asteroid 253 Mathilde from tracking data during the NEAR flyby. Science 279, 2106-2109. 